\title{
Theoretical Examination of Two Opposite Mechanisms proposed for Hepatitis Delta Virus ribozyme
}

\author{
Kai Wei, Lei Liu*, Yu-Hui Cheng, Yao Fu, Qing-Xiang Guo* \\ Department of Chemistry, University of Science and Technology of \\ China, Hefei 230026, China
}

Supporting Information

\section{Computational details}

General. All the MD simulations were accomplished with the NAMD package ${ }^{1}$, and force field parameters used in these simulations are from the parm99 all atom force-field parameter $\operatorname{set}^{2}$. Quantum mechanical calculations were carried out using Gaussian03 program ${ }^{3}$. And the data of charges analysis is Mulliken atomic charges. All the calculations were performed on the HP Superdome supercomputer of USTC-HP Laboratory of High Performance Computing and on workstations in our laboratory.

Preparation of the HDV Ribozyme System. The initial structure was chosen from X-ray data (PDB code: 1SJ3). The 1SJ3 structure is C75U mutant HDV genomic ribozyme precursor with $\mathrm{Mg}^{2+}$ bound. For the 1SJ3 structure, we mutated $\mathrm{C} 75 \mathrm{U}$ back to $\mathrm{C} 75$. And hydrogens for heavy atoms were added by Psfgen in NAMD package. After relaxing the added atoms using NAMD package in the gas phase, the structure was immersed in a cubic TIP3P water box $\left(70 \times 110 \times 70 \AA^{3}\right)$ and neutralized by addition of $68 \mathrm{Na}^{+}$counterions. This led to a 1SJ3 simulations system of 46770 atoms. The system was then subjected to a standard 9-step MD protocol equilibration process which includes energy 
minimization and partially restrained molecular dynamics simulation ${ }^{4}$ (see Figure 1). The final equilibrated structure was the starting point of the free MD procedure. Molecular dynamics simulations with periodic boundary conditions were conducted. And timestep was set as $1.0 \mathrm{fs}$. A cutoff radius of $12 \AA$ was introduced for nonbonding interaction, updating the neighbor pair list every 10 steps. The electrostatic interactions were calculated with the Particle Mesh Ewald method ${ }^{5}$. The SHAKE algorithm ${ }^{6}$ was used to constrain all bond lengths involving hydrogens. And simulations were carried out in the isothermic -isobaric ensemble (NPT) for $T=300 \mathrm{~K}$ and $P=1 \mathrm{~atm}$.

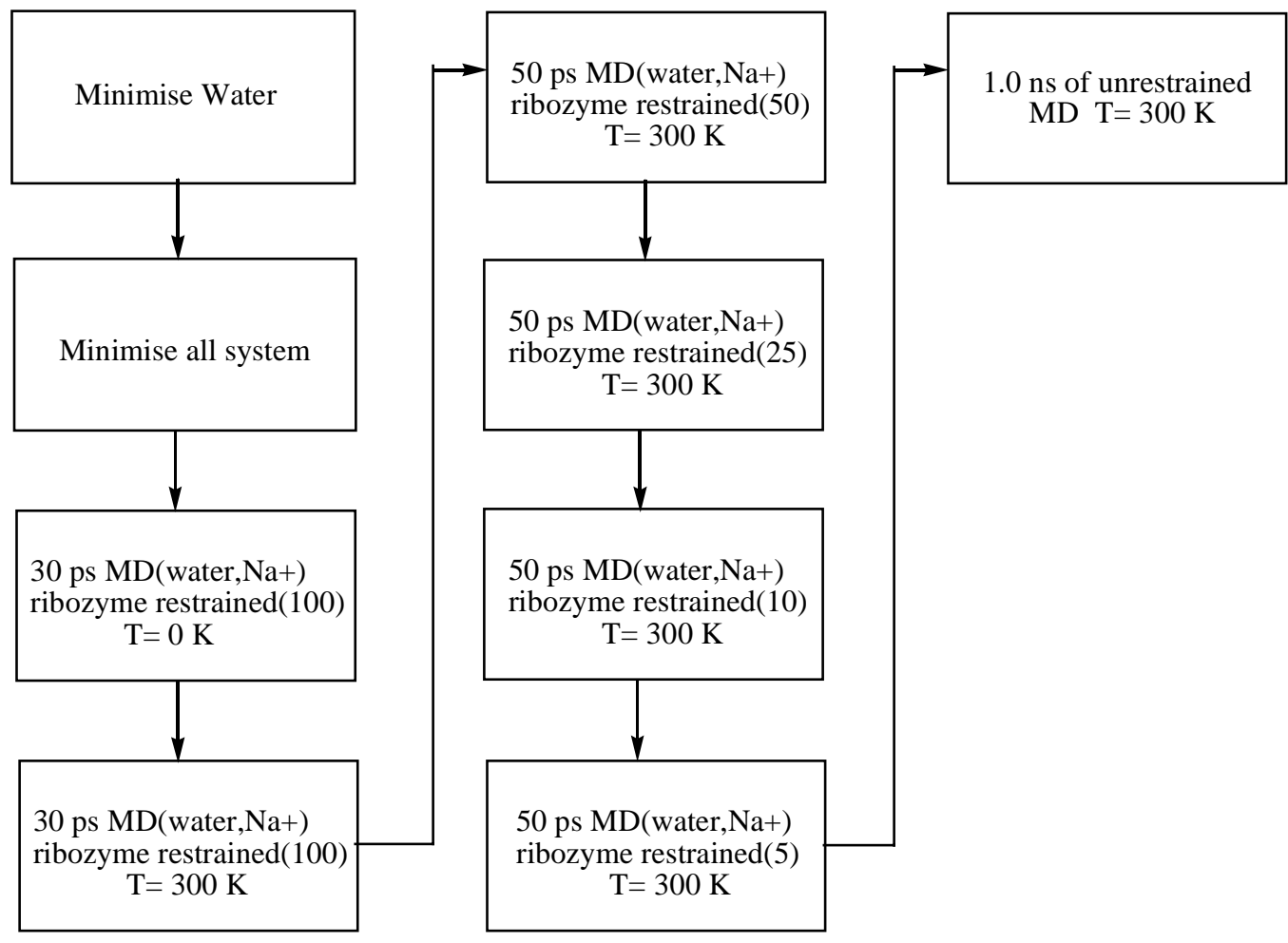

Figure 1. The 9-step MD protocol equilibration process for the HDV ribozyme.

From the free MD simulation trajectory we chose two frames of them which have the similar conformations comparing with the two mechanisms mentioned above respectively as the initial conformations for the two mechanisms hypothesis ${ }^{7,8}$. And the QM models were obtained by deleting the atoms except indicating in Figure 2. And we use hydrogen atoms to saturate the QM model 
system. For the QM model system of the two mechanisms, they both have 83 atoms in which there are 42 non-hydrogen atoms. And also they have the same overall charge (i.e. -1 e) and the spin multiplicity (i.e. 1).

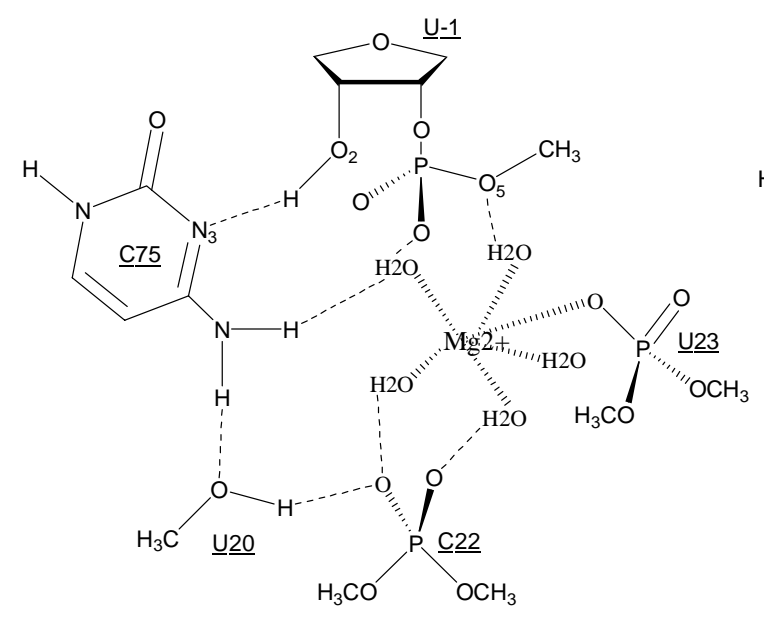

I (C75-base mechanism)

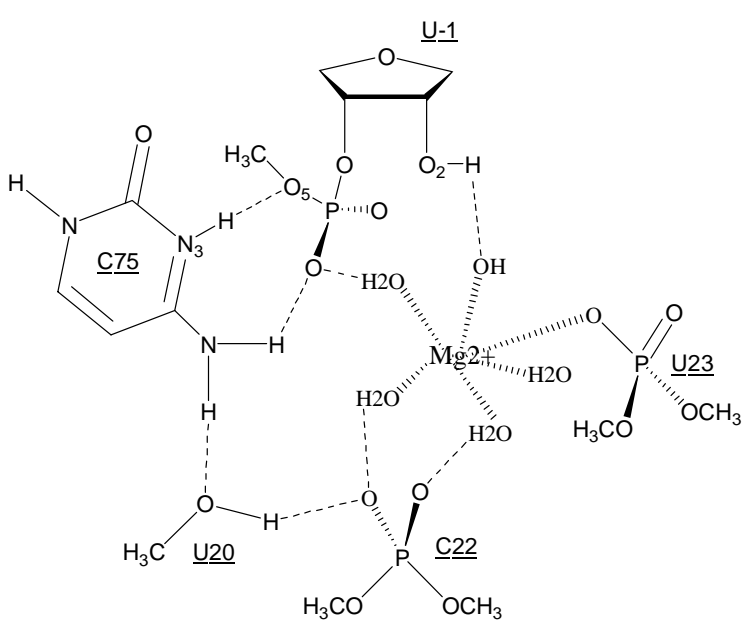

II (C75-acid mechanism)

Figure 2. The selected QM models from the MD procedure for the two mechanisms hypothesis

QM calculations. All geometry optimization and single-point energy calculation in this study were performed using B3LYP density functional theory $^{9-11}$ implemented in the Gaussian03 program package. Geometry optimization and frequency analysis for all the above QM models were at the b3lyp/3-219* level followed by single-point energy calculations using the b3lyp/6-31+g* basis set. Frequency analysis indicated that the located stationary points were correct, such as no imaginary frequencies existed for minima and only one clear imaginary frequency existed for transition states. The effect of solvent was modeled with the polarizable continuum models (PCM) developed by Tomasi and co-workers ${ }^{12}$ at the b3lyp/6-31 $+g^{*}$ level. All energies discussed below included the zero-point energy (ZPE) correction, temperature correction and salvation effects. Typical errors reported for B3LYP are about $2-3 \mathrm{kcal} / \mathrm{mol}$ for reaction barriers and energies for complexes ${ }^{13,14}$. And for the 
reaction pathway searching procedure, we first used Opt=Modredundant option to do the potential energy surface scan. And then we chose the saddle point structure to do the TS analysis. Later we did the IRC analysis to conform the TS structure.

NAC procedure. Firstly, we put the models of the two mechanisms treated in QM calculation procedure back into the two selected frames which were mentioned in the final part of "Preparation of the HDV Ribozyme System." section, respectively. And then we used NAMD program to run $100 \mathrm{ps}$ free MD for the two systems, respectively. Time step was set as $1.0 \mathrm{fs}$. A cutoff radius of $12 \AA$ was introduced for nonbonding interaction, updating the neighbor pair list every 10 steps. The electrostatic interactions were calculated with the Particle Mesh Ewald method. The SHAKE algorithm was used to constrain all bond lengths involving hydrogens. And simulations were carried out in the isothermic -isobaric ensemble (NPT) for $T=300 \mathrm{~K}$ and $P=1 \mathrm{~atm}$. After we got the trajectories of each simulation, we could perform the next step.

Secondly, we used the concept of near-attack conformation (NAC) to get the probabilities of the near-attack conformations ${ }^{15}$. The NAC had to meet two criteria:

(1). For C75-base mechanism, the distance between $\mathrm{N}_{3}$ atom of general base $\mathrm{C} 75$ and $\mathrm{O}_{2}^{\prime}$ atom of $\mathrm{U}-1$ residue should be within $2.698 \pm 0.4 \AA(2.698 \AA$ was the distance of $\mathrm{N}_{3}$ atom of general base $\mathrm{C} 75$ and $\mathrm{O}_{2}$, of $\mathrm{U}-1$ residue of the reactant in model system which was treated in "QM calculations." section and the similar values below is also from model system) and synchronously the distance between $\mathrm{O}$ atom of general acid water coordinated with $\mathrm{Mg}^{2+}$ and the leaving group $\mathrm{O}_{5}$ atom of $\mathrm{G} 1$ residue (see Figure 2 ) should be within $2.614 \pm 0.4$ $\AA$.

(2). For C75-acid mechanism, the distance between $\mathrm{N}_{3}$ atom of general acid $\mathrm{C} 75$ and $\mathrm{O}_{5}^{\prime}$ atom of $\mathrm{G} 1$ residue should be within $2.623 \pm 0.4 \AA$ and synchronously the distance between $O$ atom of general base a hydrated 
magnesium hydroxide, $[\mathrm{Mg}(\mathrm{OH})]^{+}$and $\mathrm{O}_{2}$ atom of $\mathrm{U}-1$ residue (see Figure 2) should be within $2.543 \pm 0.4 \AA$.

And from the free MD trajectories, we could calculate the probabilities of the NAC of the two mechanisms according to the criteria mentioned above. And we got that the probabilities of the NAC were $55.5 \%$ and $0.1 \%$ for $\mathrm{C} 75$-base mechanism and $\mathrm{C} 75$-acid mechanism, respectively.

$\mathrm{p} K_{\mathrm{a}}$ calculations. For better understanding the mechanism of the self-cleaving reaction of HDV ribozyme, we calculated the $\mathrm{p} K_{\mathrm{a}}$ of $\mathrm{N} 3$ of $\mathrm{C} 75$ and water coordinated with $\mathrm{Mg}^{2+}$. Firstly, we put the models of the two mechanisms treated in QM calculation procedure back into the two selected frames which were mentioned in the final part of "Preparation of the HDV Ribozyme System." section, respectively. And then we used QMMM +FEP ${ }^{16}$. method to obtain these values based on equation $1^{17}$ :

$$
\mathrm{BH}^{+}+\mathrm{H}_{2} \mathrm{O} \rightleftharpoons \mathrm{B}+\mathrm{H}_{3} \mathrm{O}^{+}
$$

in which $\mathrm{BH}^{+}$is the initial structure for $\mathrm{C} 75$-base and $\mathrm{C} 75$-acid mechanisms which were obtained by the method described above. In the QMMM calculation, the system was partitioned into a QM subsystem (see Figure 3) and a MM subsystem.

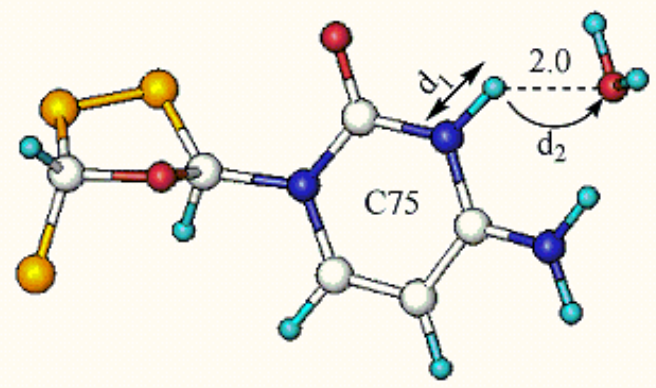

pKa calculation of $\mathrm{C} 75$

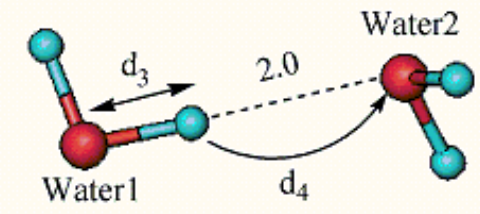

pKa calculation of water coordinated with $\mathrm{Mg}^{2+}$

Figure 3. QM parts of $\mathrm{QMMM}$ calculations for the two mechanisms hypothesis. And white atom is $\mathrm{C}$ atom, red atom is $\mathrm{O}$ atom, yellow atom is $\mathrm{F}$ atom, blue atom is $\mathrm{N}$ atom and green atom is $\mathrm{H}$ atom.

And the $\mathrm{QM} / \mathrm{MM}$ model was obtained by deleting the water molecule more 
than $27.0 \AA$ away from the $\mathrm{Mg}^{2+}$ divalent cation which is the active site of this reaction. For each prepared QM/MM system an iterative optimization procedure ${ }^{16}$ was applied to the system with B3lyp/3-21g basis set QM/MM calculations, leading to an optimized structure for the reactant. We then employed the reaction coordinate driving method ${ }^{16}$ and used $R C=d_{1}-d_{2}$ as the reaction coordinate for pKa calculation of $\mathrm{C} 75$ and $\mathrm{RC}=\mathrm{d}_{3}-\mathrm{d}_{4}$ as the reaction coordinate for $\mathrm{pKa}$ calculation of water coordinated with $\mathrm{Mg}^{2+}$ to drive these reactions. And then we use $6-31 \mathrm{~g}$ (basis set to generate the single point energy. In the FEP method the QM part is frozen and just run MD for the MM partition to get the free energy change of the deprotonated reaction. The calculations were carried out using modified versions of the Gaussian98 and TINKER programs ${ }^{18}$. For the QM subsystem, criteria used for geometry optimizations follow Gaussian98 defaults. For the MM subsystem, the convergence criterion used is to have the root-mean- square (RMS) energy gradient be less than $0.1 \mathrm{kcal}$ mol-1 $\AA-1$. In the MM minimizations, only atoms within $20 \AA$ of the $\mathrm{Mg}^{2+}$ divalent cation were allowed to move. No cutoff for nonbonding interactions was used in the QM/MM calculations and the MM minimizations.

And we got that the $\mathrm{pKa}$ of $\mathrm{C} 75$ is 6.4 and that of water coordinated with $\mathrm{Mg}^{2+}$ is 9.2 .

\section{Reference:}

1. Canto, J.; Haro, I.; Alsina, M. A.; Perez, J. J., J. Phys. Chem. B 2003, 107, 6603-6608.

2. Phillips, J. C.; Braun, R.; Wang, W.; Gumbart, J.; Tajkhorshid, E.; Villa, E.; Chipot, C.; Skeel, R. D.; Kale, L.; K., S., J. Comp. Chem. 2005, 26, 1781-1802.

3. Cornell, W. D.; Cieplak, P.; Bayly, C. I.; Gould, I. R.; Merz, K. M.; Ferguson, D. M.; Spellmeyer, D. C.; Fox, T.; Caldwell, J. W.; Kollman, P. A., J. Am. Chem. Soc. 1995, 117, 5179-5197.

4. Frisch, M. J.; Trucks, G. W., et al., GAUSSIAN 03, R. A.; Gaussian, I., Pittsburgh, PA, 2003.

5. Shields, G. C.; Laughton, C. A.; Orozco, M., J. Am. Chem. Soc. 1998, 120, 5895-5904.

6. Darden, T.; York, D.; Pedersen, L., J. Chem. Phys. 1993, 98, 10089-10092.

7. Ke, A.; Zhou, K.; Ding, F.; Cate, J. H. D.; Doudna, J. A., Nature 2004, 429, (201-205).

8. Luptak, A.; Ferre-DrAmare, A. R.; Zhou, K.; Zilm, K. W.; Doudna, J. A., J. Am. Chem. Soc. 2001, $1213,8447-8452$. 
9. Ryekaert, J. P.; Ciccotti, G.; Berendsen, H. J. C., J. Comp. Phys. 1977, 23, 327-341.

10. Becke, A. D., J. Chem. Phys. 1986, 84, 4524-4529.

11. Becke, A. D., Phys. Rev. A At. Mol. Opt. Phys. 1988, 38, 3098-3100.

12. Lee, C.; Yang, W.; Parr, R. G., Phys. Rev. B 1988, 37, 785-789.

13. Barone, V.; Cossi, M.; Tomasi, J., J. Chem. Phys. 1997, 107, 3210-3221.

14. Lynch, B. J.; Truhlar, D. G., J. Phys. Chem. A 2001, 105, 2936-2941.

15. Felice C. Lightstone; Thomas C. Bruice, J. Am. Chem. Soc. 1994, 116, 10789-10790.

16. Zhang, Y.; Liu H.; Yang W., J. Chem. Phys. 2000, 112, 3483-3492..

17. da Silva, E. F.; Svendsen, H. F. Ind. Eng. Chem. Res. 2003, 42, 4414-4421.

18. Ponder, J. W. TINKER, Software Tools for Molecular Design, version 3.6; The most updated version for the TINKER program can be obtained from J. W. Ponder's World Wide Web site at http://dasher.wustl.edu/tinker.,February 1998.

\section{Geometry Summaries}


Cartesian coordinates and distances are reported in angstroms. Electronic energies are reported in Hartrees. Charge is specified for each structure.

\section{Reactant of C75-base mechanism}

b3lyp/3-21g* optimized structure:

$\begin{array}{cccc}\mathrm{C} & 0.000000 & 0.000000 & 0.000000 \\ \mathrm{~N} & 0.000000 & 0.000000 & 1.372268 \\ \mathrm{C} & 1.160290 & 0.000000 & 2.073277 \\ \mathrm{C} & 2.451297 & 0.081824 & 1.405555 \\ \mathrm{C} & 2.457554 & 0.081444 & 0.057453 \\ \mathrm{~N} & 1.276515 & 0.006040 & -0.641969 \\ \mathrm{~N} & 1.164467 & -0.070925 & 3.394731 \\ \mathrm{O} & -1.014765 & -0.001293 & -0.725617 \\ \mathrm{O} & 3.643174 & 0.353995 & 4.327694 \\ \mathrm{C} & 4.232102 & -0.741895 & 5.115322 \\ \mathrm{O} & 3.280517 & 2.142920 & 6.225469 \\ \mathrm{P} & 3.995002 & 1.709329 & 7.514413 \\ \mathrm{O} & 5.583672 & 1.629187 & 7.187050 \\ \mathrm{C} & 6.211933 & 2.849062 & 6.646659 \\ \mathrm{O} & 3.584934 & 0.403152 & 8.153847 \\ \mathrm{O} & 3.922683 & 2.920309 & 8.575197 \\ \mathrm{C} & 3.178857 & 2.701257 & 9.841172 \\ \mathrm{O} & 1.487131 & -0.590084 & 7.158722 \\ \mathrm{Mg} & -0.131720 & 0.634664 & 7.258999 \\ \mathrm{O} & -0.986227 & -1.138349 & 8.140144 \\ \mathrm{O} & -0.812637 & 0.314623 & 5.261104 \\ \mathrm{O} & 0.338538 & 1.177020 & 9.228148 \\ \mathrm{P} & -0.825228 & 1.412958 & 10.180570 \\ \mathrm{O} & -1.996286 & 0.428135 & 9.963702 \\ \mathrm{O} & 0.697095 & 2.354626 & 6.623675 \\ \mathrm{O} & -2.101199 & 1.245422 & 7.505877 \\ \mathrm{O} & -0.377175 & 1.333509 & 11.728121 \\ \mathrm{C} & -0.524363 & 0.050810 & 12.436946 \\ \mathrm{O} & -1.321112 & 2.932846 & 10.116322 \\ \mathrm{C} & -0.991441 & 3.815476 & 8.961972 \\ \mathrm{O} & -1.082247 & 2.799847 & 4.828462 \\ \mathrm{P} & -2.581112 & 2.991835 & 4.612941 \\ \mathrm{O} & -3.228594 & 3.975185 & 3.707013 \\ \mathrm{C} & -3.261186 & 1.484497 & 4.517728 \\ \mathrm{O} & -3.905109 & 1.008930 & 3.292550 \\ & -4.031047 & -0.763251 & 1.746414\end{array}$




\begin{tabular}{|c|c|c|c|}
\hline $\mathrm{C}$ & -3.669456 & 0.460728 & 0.958382 \\
\hline$C$ & -3.285185 & 1.562557 & 1.976273 \\
\hline $\mathrm{O}$ & -1.879127 & 1.752025 & 2.195187 \\
\hline ) & -3.107777 & 3.271202 & 6.196458 \\
\hline$\tau$ & -4.550991 & 3.556894 & 6.330816 \\
\hline H & -4.253767 & -1.122715 & 3.784219 \\
\hline H & -2.835430 & 0.245214 & 0.285299 \\
\hline & -4.979576 & 1.213453 & 3.327055 \\
\hline $\mathrm{H}$ & -3.708862 & 2.536926 & 1.734619 \\
\hline $\mathrm{H}$ & -1.296056 & 0.942542 & 1.934177 \\
\hline$\theta$ & -2.576450 & -0.668480 & 3.310469 \\
\hline $\mathrm{H}$ & -4.562766 & 0.744344 & 0.391636 \\
\hline $\mathrm{H}$ & -5.126702 & 2.635492 & 6.193836 \\
\hline H & -4.827708 & 4.278728 & 5.559649 \\
\hline $\mathrm{H}$ & -4.710686 & 3.964771 & 7.329781 \\
\hline $\mathrm{H}$ & 4.481982 & -1.543365 & 4.415096 \\
\hline $\mathrm{H}$ & 5.139950 & -0.398061 & 5.624682 \\
\hline $\mathrm{H}$ & 3.505924 & 1.142788 & 4.980392 \\
\hline $\mathrm{H}$ & 3.524750 & -1.106166 & 5.865596 \\
\hline $\mathrm{H}$ & 7.243141 & 2.589428 & 6.403869 \\
\hline $\mathrm{H}$ & 6.183542 & 3.645298 & 7.397055 \\
\hline $\mathrm{H}$ & 5.677925 & 3.180126 & 5.750635 \\
\hline $\mathrm{H}$ & 3.725499 & 2.004958 & 10.483396 \\
\hline $\mathrm{H}$ & 2.183856 & 2.297442 & 9.644154 \\
\hline $\mathrm{H}$ & -0.846176 & 4.813198 & 9.378438 \\
\hline $\mathrm{H}$ & 3.115404 & 3.681562 & 10.315828 \\
\hline $\mathrm{H}$ & -1.820730 & 3.811235 & 8.251855 \\
\hline $\mathrm{H}$ & -0.099108 & 3.464438 & 8.438375 \\
\hline $\mathrm{H}$ & 0.337544 & -0.594561 & 12.235815 \\
\hline $\mathrm{H}$ & -1.439743 & -0.447124 & 12.109238 \\
\hline $\mathrm{H}$ & -0.568283 & 0.285408 & 13.501657 \\
\hline $\mathrm{H}$ & 3.368624 & 0.141830 & -0.524993 \\
\hline $\mathrm{H}$ & 3.335982 & 0.167193 & 2.019601 \\
\hline $\mathrm{H}$ & 0.301770 & -0.105526 & 3.958287 \\
\hline $\mathrm{H}$ & 2.090605 & 0.025354 & 3.879560 \\
\hline $\mathrm{H}$ & 1.251228 & 0.013354 & -1.655449 \\
\hline $\mathrm{H}$ & -1.798127 & 0.167885 & 5.222088 \\
\hline $\mathrm{H}$ & -0.773138 & 1.324898 & 4.934329 \\
\hline $\mathrm{H}$ & 0.123554 & 2.805152 & 5.917872 \\
\hline $\mathrm{H}$ & 1.699947 & 2.370304 & 6.403939 \\
\hline $\mathrm{H}$ & 1.124542 & -1.358365 & 7.668929 \\
\hline $\mathrm{H}$ & 2.360910 & -0.223451 & 7.631175 \\
\hline $\mathrm{H}$ & -2.536622 & 2.062409 & 7.081605 \\
\hline $\mathrm{H}$ & -2.443009 & 1.030212 & 8.431478 \\
\hline
\end{tabular}




$\begin{array}{llll}\mathrm{H} & -1.424480 & -0.674563 & 8.967511 \\ \mathrm{H} & -1.728674 & -1.418413 & 7.546138\end{array}$

$\mathrm{E}(\mathrm{RB}+\mathrm{HF}-\mathrm{LYP})=-3526.9540208$ (single point energy, and the values below have the same definition)

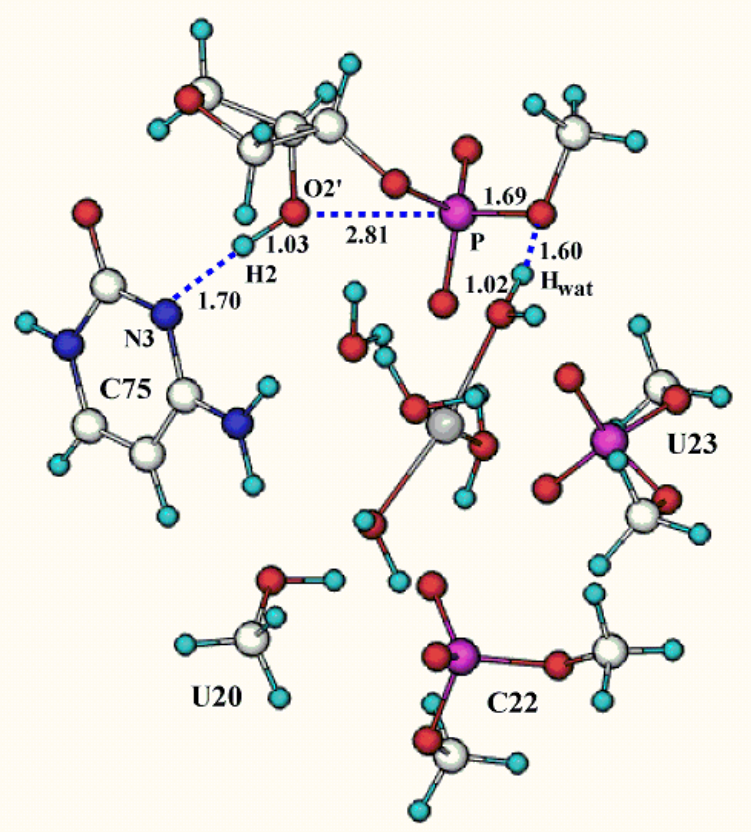

Figure 4. Optimized structure of the Reactant of C75-base mechanism.

\section{TS1 of C75-base mechanism}

b3lyp/3-21g* optimized structure:

$\begin{array}{lrrr}\mathrm{C} & 0.000000 & 0.000000 & 0.000000 \\ \mathrm{~N} & 0.000000 & 0.000000 & 1.366527 \\ \mathrm{C} & 1.153120 & 0.000000 & 2.130137 \\ \mathrm{~N} & 2.359271 & -0.122270 & 1.402506 \\ \mathrm{C} & 2.397101 & -0.220371 & 0.027771 \\ \mathrm{C} & 1.266816 & -0.155058 & -0.700807 \\ \mathrm{O} & 1.169244 & 0.110142 & 3.364803 \\ \mathrm{~N} & -1.088021 & 0.153066 & -0.714318 \\ \mathrm{O} & -1.939995 & -0.626661 & 2.810164\end{array}$




$\begin{array}{cccc}\mathrm{C} & -2.020152 & -0.294106 & 4.208191 \\ \mathrm{C} & -3.561193 & -0.318767 & 4.362728 \\ \mathrm{C} & -3.919523 & 1.078829 & 3.814493 \\ \mathrm{O} & -2.862484 & 1.982169 & 4.279900 \\ \mathrm{C} & -1.648619 & 1.157880 & 4.515278 \\ \mathrm{O} & -4.117386 & -1.355712 & 3.512640 \\ \mathrm{P} & -3.059851 & -2.251191 & 2.556839 \\ \mathrm{O} & -2.904796 & -1.856492 & 1.035656 \\ \mathrm{O} & -4.464340 & -3.224109 & 2.161129 \\ \mathrm{C} & -5.041232 & -3.990864 & 3.268916 \\ \mathrm{O} & -2.157579 & -3.195914 & 3.282658 \\ \mathrm{O} & -3.650727 & 0.202717 & 0.041641 \\ \mathrm{Mg} & -4.960074 & -0.901241 & -1.193917 \\ \mathrm{O} & -6.257724 & -2.074561 & -2.377671 \\ \mathrm{P} & -7.477126 & -2.658616 & -1.675399 \\ \mathrm{O} & -8.617945 & -3.106855 & -2.731391 \\ \mathrm{C} & -9.730853 & -2.183964 & -2.999873 \\ \mathrm{O} & -5.776712 & -1.672509 & 0.563525 \\ \mathrm{O} & -4.484081 & 0.190051 & -2.854661 \\ \mathrm{O} & -3.561758 & -2.333626 & -1.343766 \\ \mathrm{O} & -6.621402 & 0.411983 & -0.923728 \\ \mathrm{O} & -8.117423 & -1.694415 & -0.656195 \\ \mathrm{O} & -7.149038 & -4.078971 & -1.018692 \\ \mathrm{C} & -5.752594 & -4.517843 & -0.735388 \\ \mathrm{O} & -3.959444 & -1.193523 & -4.897528 \\ \mathrm{P} & -2.965865 & -2.323389 & -4.782393 \\ \mathrm{O} & -3.682447 & -3.751712 & -4.977372 \\ \mathrm{C} & -5.166401 & -3.807418 & -4.917682 \\ \mathrm{O} & -2.068130 & -2.394655 & -3.537585 \\ \mathrm{O} & -1.986024 & -2.243385 & -6.077063 \\ \mathrm{C} & -1.033548 & -3.352383 & -6.259464 \\ \mathrm{O} & -0.586881 & -0.302545 & -3.259580 \\ \mathrm{C} & -1.254283 & 0.610105 & -4.207105 \\ \mathrm{H} & -6.770057 & -1.795296 & 0.512254 \\ \mathrm{H} & -5.315314 & -2.330788 & 1.218315 \\ \mathrm{H} & -4.216631 & -4.448737 & 3.822976 \\ \mathrm{H} & -5.689701 & -4.762777 & 2.844881 \\ \mathrm{H} & -0.459767 & 5.393506 \\ \mathrm{H} & -1.460020 & 4.171425 \\ \mathrm{H} & -1.024062 & 4.828576 \\ \mathrm{H} & -3.578914 & 3.892050\end{array}$




\begin{tabular}{|c|c|c|c|}
\hline $\mathrm{H}$ & -3.923112 & 0.985964 & 2.723232 \\
\hline $\mathrm{H}$ & 3.204259 & -0.146953 & 1.961927 \\
\hline $\mathrm{H}$ & 3.374304 & -0.341529 & -0.421371 \\
\hline $\mathrm{H}$ & 1.232070 & -0.221740 & -1.778765 \\
\hline $\mathrm{H}$ & -2.066542 & 0.265981 & -0.337945 \\
\hline $\mathrm{H}$ & -0.974456 & 0.046839 & -1.766883 \\
\hline $\mathrm{H}$ & -1.081378 & -1.217047 & -3.354896 \\
\hline $\mathrm{H}$ & -0.697684 & 1.551085 & -4.201525 \\
\hline $\mathrm{H}$ & -2.295967 & 0.786018 & -3.918153 \\
\hline $\mathrm{H}$ & -1.238974 & 0.175891 & -5.213584 \\
\hline $\mathrm{H}$ & -0.398314 & -3.455848 & -5.373642 \\
\hline $\mathrm{H}$ & -0.429088 & -3.106904 & -7.133912 \\
\hline $\mathrm{H}$ & -1.578016 & -4.288016 & -6.419882 \\
\hline $\mathrm{H}$ & -5.540030 & -3.293173 & -4.029822 \\
\hline $\mathrm{H}$ & -5.595492 & -3.342654 & -5.810041 \\
\hline $\mathrm{H}$ & -5.417003 & -4.868668 & -4.887720 \\
\hline $\mathrm{H}$ & -4.321207 & -0.379377 & -3.731344 \\
\hline $\mathrm{H}$ & -5.267879 & 0.779043 & -2.987679 \\
\hline $\mathrm{H}$ & -3.934773 & 0.942190 & 0.632658 \\
\hline $\mathrm{H}$ & -3.287118 & -0.748169 & 0.678068 \\
\hline $\mathrm{H}$ & -3.150425 & -2.427292 & -0.408057 \\
\hline $\mathrm{H}$ & -2.906040 & -2.427815 & -2.114462 \\
\hline $\mathrm{H}$ & -6.473110 & 0.699223 & 0.014715 \\
\hline $\mathrm{H}$ & -7.363280 & -0.327383 & -0.860065 \\
\hline $\mathrm{H}$ & -5.510237 & -4.319728 & 0.309612 \\
\hline $\mathrm{H}$ & -5.033220 & -3.964077 & -1.341978 \\
\hline $\mathrm{H}$ & -5.725610 & -5.587910 & -0.947477 \\
\hline $\mathrm{H}$ & -9.972431 & -1.626064 & -2.092301 \\
\hline $\mathrm{H}$ & -9.460898 & -1.484629 & -3.799348 \\
\hline $\mathrm{H}$ & -10.578641 & -2.793650 & -3.318745 \\
\hline
\end{tabular}




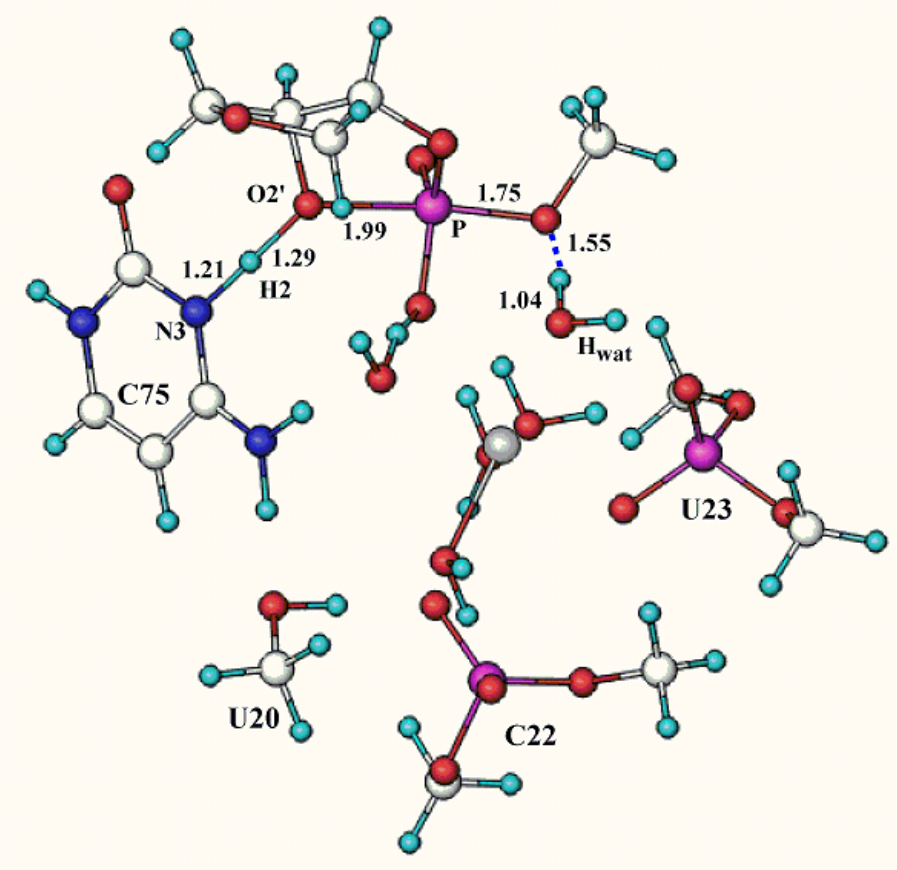

Figure 5. Optimized structure of the Ts1 of C75-base mechanism.

\section{Intermediate of C75-base mechanism}

b31yp/3-21g* optimized structure:

$\begin{array}{lrrr}\mathrm{C} & 0.000000 & 0.000000 & 0.000000 \\ \mathrm{~N} & 0.000000 & 0.000000 & 1.386031 \\ \mathrm{C} & 1.138393 & 0.000000 & 2.148435 \\ \mathrm{C} & 2.419267 & 0.168901 & 1.477248 \\ \mathrm{C} & 2.436766 & 0.240657 & 0.133528 \\ \mathrm{~N} & 1.271628 & 0.135061 & -0.597103 \\ \mathrm{~N} & 1.133070 & -0.165661 & 3.445549 \\ \mathrm{O} & -1.017631 & -0.122282 & -0.694779 \\ \mathrm{O} & -2.315385 & 0.613309 & 2.172345 \\ \mathrm{P} & -2.716906 & 2.164847 & 3.244498 \\ \mathrm{O} & -1.340318 & 1.798992 & 3.946427 \\ \mathrm{C} & -3.532586 & 0.228043 & 1.503447 \\ \mathrm{C} & -3.525620 & -1.221914 & 1.020390 \\ \mathrm{O} & -3.922074 & -2.071574 & 2.172679 \\ \mathrm{C} & -4.144292 & -1.181398 & 3.317317\end{array}$




\begin{tabular}{|c|c|c|c|}
\hline $\mathrm{C}$ & -4.479412 & 0.206096 & 2.728198 \\
\hline $\mathrm{O}$ & -4.088401 & 1.261120 & 3.644029 \\
\hline $\mathrm{O}$ & -3.149427 & 3.127633 & 4.663157 \\
\hline $\mathrm{C}$ & -4.396094 & 3.889194 & 4.557519 \\
\hline $\mathrm{O}$ & -2.861459 & 3.145232 & 2.123980 \\
\hline $\mathrm{O}$ & -0.889288 & -0.237579 & 5.147291 \\
\hline $\mathrm{Mg}$ & -0.588425 & 0.886386 & 6.905633 \\
\hline $\mathrm{O}$ & 0.278742 & 2.320570 & 5.800588 \\
\hline $\mathrm{O}$ & -1.686125 & -0.439616 & 8.174878 \\
\hline $\mathrm{O}$ & -0.314930 & 2.080901 & 8.626048 \\
\hline $\mathrm{P}$ & -1.576474 & 2.646630 & 9.266578 \\
\hline $\mathrm{O}$ & -2.759948 & 1.658671 & 9.266715 \\
\hline $\mathrm{O}$ & -2.519972 & 1.606987 & 6.631433 \\
\hline $\mathrm{O}$ & 1.080282 & -0.177391 & 7.426983 \\
\hline $\mathrm{O}$ & -1.978560 & 4.052614 & 8.620263 \\
\hline $\mathrm{C}$ & -1.463399 & 4.486006 & 7.289565 \\
\hline $\mathrm{O}$ & -1.311370 & 3.116973 & 10.792241 \\
\hline $\mathrm{C}$ & -1.662061 & 2.195108 & 11.882782 \\
\hline $\mathrm{O}$ & 3.052906 & 1.244021 & 8.104572 \\
\hline $\mathrm{P}$ & 3.485075 & 2.369976 & 7.198023 \\
\hline $\mathrm{O}$ & 2.935840 & 2.416982 & 5.763502 \\
\hline $\mathrm{O}$ & 5.106228 & 2.310851 & 7.087969 \\
\hline $\mathrm{C}$ & 5.765188 & 3.421831 & 6.379691 \\
\hline $\mathrm{O}$ & 3.236219 & 3.803293 & 7.886784 \\
\hline $\mathrm{C}$ & 2.372918 & 3.861846 & 9.095383 \\
\hline $\mathrm{O}$ & 3.531749 & 0.312040 & 4.407900 \\
\hline $\mathrm{C}$ & 3.982172 & -0.581574 & 5.492178 \\
\hline $\mathrm{H}$ & -3.018583 & 1.735837 & 7.490231 \\
\hline $\mathrm{H}$ & -2.829010 & 2.253252 & 5.873622 \\
\hline $\mathrm{H}$ & -1.051643 & 0.218923 & 1.808280 \\
\hline $\mathrm{H}$ & -4.418625 & 4.350441 & 3.566073 \\
\hline $\mathrm{H}$ & -5.265062 & 3.233153 & 4.673154 \\
\hline $\mathrm{H}$ & -4.392304 & 4.658486 & 5.335483 \\
\hline $\mathrm{H}$ & -5.536432 & 0.305213 & 2.469570 \\
\hline $\mathrm{H}$ & -4.943514 & -1.604007 & 3.930908 \\
\hline $\mathrm{H}$ & -3.812726 & 0.949309 & 0.730460 \\
\hline $\mathrm{H}$ & -4.270089 & -1.356613 & 0.225661 \\
\hline $\mathrm{H}$ & -2.542439 & -1.495058 & 0.638501 \\
\hline $\mathrm{H}$ & -3.233122 & -1.049838 & 3.910345 \\
\hline $\mathrm{H}$ & 1.274318 & 0.160231 & -1.610474 \\
\hline $\mathrm{H}$ & 3.350289 & 0.371830 & -0.431587 \\
\hline $\mathrm{H}$ & 3.297489 & 0.239081 & 2.103039 \\
\hline $\mathrm{H}$ & 0.278372 & -0.290440 & 4.063150 \\
\hline $\mathrm{H}$ & 2.076930 & -0.054701 & 3.929193 \\
\hline
\end{tabular}




$\begin{array}{rrrr}\mathrm{H} & 3.333040 & 1.232068 & 4.863549 \\ \mathrm{H} & 4.297132 & -1.521863 & 5.031773 \\ \mathrm{H} & 3.176416 & -0.763565 & 6.210987 \\ \mathrm{H} & 4.827254 & -0.125439 & 6.020714 \\ \mathrm{H} & 5.371018 & 3.509153 & 5.361866 \\ \mathrm{H} & 6.831293 & 3.191197 & 6.355852 \\ \mathrm{H} & 5.587655 & 4.360721 & 6.913092 \\ \mathrm{H} & 1.435509 & 3.326860 & 8.929953 \\ \mathrm{H} & 2.893927 & 3.420623 & 9.950000 \\ \mathrm{H} & 2.190511 & 4.922743 & 9.271806 \\ \mathrm{H} & 1.889561 & 0.408031 & 7.773024 \\ \mathrm{H} & 0.758744 & -0.759090 & 8.160135 \\ \mathrm{H} & -1.528738 & -0.988271 & 5.081529 \\ \mathrm{H} & -1.244519 & 0.724482 & 4.471691 \\ \mathrm{H} & -0.280036 & 2.400278 & 4.943236 \\ \mathrm{H} & 1.280542 & 2.429588 & 5.675922 \\ \mathrm{H} & -2.389206 & -0.740109 & 7.541827 \\ \mathrm{H} & -2.153882 & 0.299001 & 8.755521 \\ \mathrm{H} & -2.186040 & 4.231930 & 6.513234 \\ \mathrm{H} & -0.534520 & 3.969953 & 7.040056 \\ \mathrm{H} & -1.324606 & 5.565947 & 7.360087 \\ \mathrm{H} & -2.546009 & 1.616627 & 11.604657 \\ \mathrm{H} & -0.828358 & 1.514138 & 12.088800 \\ \mathrm{H} & -1.859856 & 2.807629 & 12.764758 \\ & & & \\ \mathrm{E}(\mathrm{RB}+\mathrm{HF}-\mathrm{LYP})=-3526.9128607 & \\ & & & \end{array}$




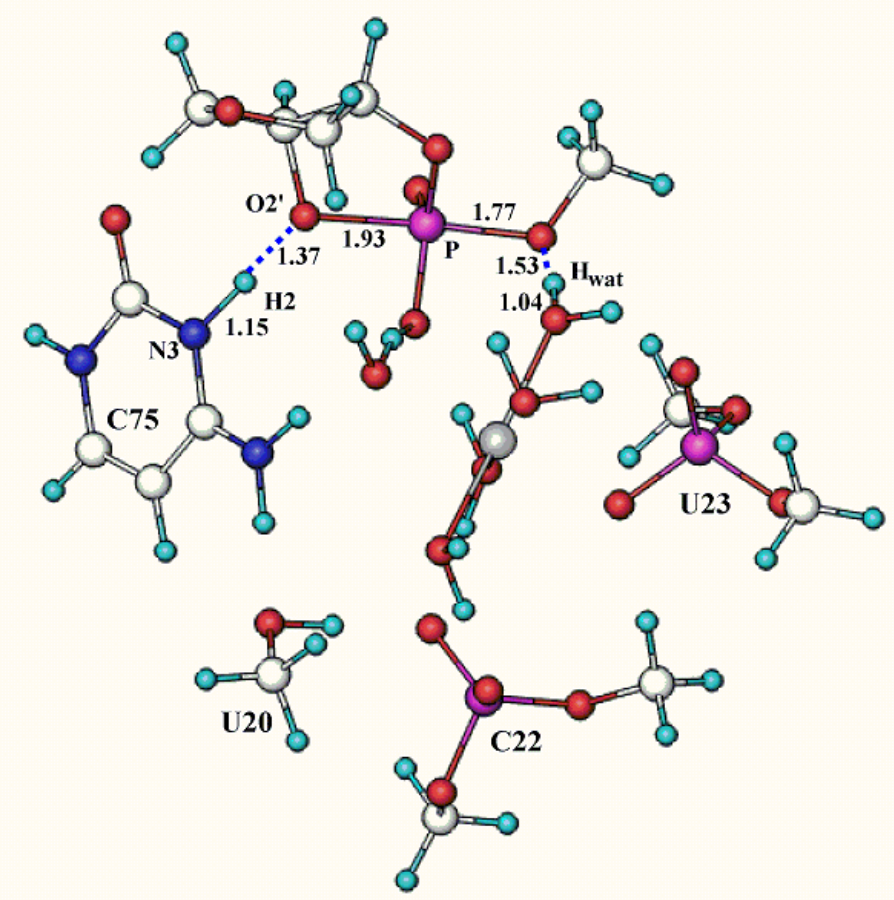

Figure 6. Optimized structure of the Intermediate of C75-base mechanism.

TS2 of C75-base mechanism

b3lyp/3-21g* optimized structure:

$\begin{array}{cccc}\mathrm{C} & 0.000000 & 0.000000 & 0.000000 \\ \mathrm{C} & 0.000000 & 0.000000 & 1.548234 \\ \mathrm{C} & 1.474740 & 0.000000 & 1.915846 \\ \mathrm{O} & 2.077243 & -0.918035 & 0.935721 \\ \mathrm{C} & 1.351259 & -0.699850 & -0.341778 \\ \mathrm{O} & -0.566787 & -1.306280 & 1.843283 \\ \mathrm{P} & -1.911012 & -1.680509 & 0.759736 \\ \mathrm{O} & -3.090892 & -1.076952 & 1.443525 \\ \mathrm{O} & -1.159596 & -0.769885 & -0.437302 \\ \mathrm{O} & -1.547248 & -3.182540 & 0.656734 \\ \mathrm{O} & -3.141573 & -2.030489 & -1.034823 \\ \mathrm{C} & -3.863183 & -0.819386 & -1.408076 \\ \mathrm{O} & -1.727479 & -3.057314 & -2.773273 \\ \mathrm{Mg} & -1.190117 & -5.005956 & -2.312154 \\ \mathrm{O} & 0.075985 & -6.607970 & -2.262303\end{array}$




\begin{tabular}{|c|c|c|c|}
\hline $\mathrm{O}$ & -2.257093 & -5.266104 & -0.659555 \\
\hline $\mathrm{O}$ & -2.575546 & -5.925929 & -3.514082 \\
\hline $\mathrm{P}$ & -3.187318 & -5.036277 & -4.596590 \\
\hline $\mathrm{O}$ & -3.889488 & -5.933549 & -5.748578 \\
\hline $\mathrm{C}$ & -3.109633 & -6.270338 & -6.948595 \\
\hline $\mathrm{O}$ & -2.177602 & -4.094620 & -5.271953 \\
\hline $\mathrm{O}$ & -4.459238 & -4.258500 & -4.028455 \\
\hline $\mathrm{C}$ & -4.800292 & -4.295923 & -2.572843 \\
\hline $\mathrm{O}$ & 0.013921 & -4.803281 & -4.144811 \\
\hline $\mathrm{O}$ & 0.100442 & -3.534156 & -1.287260 \\
\hline $\mathrm{O}$ & -1.180492 & -8.801050 & -2.034462 \\
\hline $\mathrm{P}$ & -2.048496 & -8.858278 & -0.801794 \\
\hline $\mathrm{O}$ & -3.606692 & -8.992172 & -1.181093 \\
\hline $\mathrm{C}$ & -4.019382 & -8.684849 & -2.575821 \\
\hline $\mathrm{O}$ & -1.894971 & -7.749541 & 0.253421 \\
\hline $\mathrm{O}$ & -1.749895 & -10.270287 & -0.051736 \\
\hline $\mathrm{C}$ & -2.575494 & -10.579053 & 1.128782 \\
\hline $\mathrm{O}$ & 0.544348 & -7.505332 & 1.018107 \\
\hline $\mathrm{C}$ & 1.279086 & -8.437803 & 0.144458 \\
\hline $\mathrm{N}$ & 0.795995 & -3.317458 & 2.786342 \\
\hline $\mathrm{C}$ & 0.983704 & -2.956684 & 4.118889 \\
\hline $\mathrm{N}$ & 1.114962 & -4.039336 & 5.005435 \\
\hline $\mathrm{C}$ & 1.071170 & -5.356431 & 4.599211 \\
\hline $\mathrm{C}$ & 0.963655 & -5.676173 & 3.297414 \\
\hline $\mathrm{C}$ & 0.869136 & -4.604009 & 2.321179 \\
\hline $\mathrm{O}$ & 1.052238 & -1.792133 & 4.528973 \\
\hline $\mathrm{N}$ & 0.885248 & -4.921734 & 1.051254 \\
\hline $\mathrm{H}$ & -1.933991 & -2.924143 & -3.736212 \\
\hline $\mathrm{H}$ & -2.525755 & -2.424627 & -1.870471 \\
\hline $\mathrm{H}$ & 0.386653 & -2.511806 & 2.196046 \\
\hline $\mathrm{H}$ & -4.217951 & -0.394964 & -0.467419 \\
\hline $\mathrm{H}$ & -3.203471 & -0.096663 & -1.904683 \\
\hline $\mathrm{H}$ & -4.711479 & -1.056626 & -2.063899 \\
\hline $\mathrm{H}$ & -0.067318 & 1.000500 & -0.432003 \\
\hline $\mathrm{H}$ & 1.955871 & -0.071901 & -1.008591 \\
\hline $\mathrm{H}$ & -0.585019 & 0.805648 & 1.999930 \\
\hline $\mathrm{H}$ & 1.885952 & 1.018713 & 1.830391 \\
\hline $\mathrm{H}$ & 1.630596 & -0.409859 & 2.912770 \\
\hline $\mathrm{H}$ & 1.153394 & -1.676547 & -0.786522 \\
\hline $\mathrm{H}$ & 1.232807 & -3.785438 & 5.979889 \\
\hline $\mathrm{H}$ & 1.138606 & -6.103724 & 5.378540 \\
\hline $\mathrm{H}$ & 0.918629 & -6.686456 & 2.913582 \\
\hline $\mathrm{H}$ & 0.817792 & -4.253783 & 0.271067 \\
\hline $\mathrm{H}$ & 0.822010 & -5.972743 & 0.839091 \\
\hline
\end{tabular}




$\begin{array}{rrrr}\mathrm{H} & -0.475693 & -7.591328 & 0.780203 \\ \mathrm{H} & 2.303193 & -8.505104 & 0.522284 \\ \mathrm{H} & 1.272904 & -8.070461 & -0.883210 \\ \mathrm{H} & 0.806796 & -9.426510 & 0.177387 \\ \mathrm{H} & -2.509443 & -9.763995 & 1.856677 \\ \mathrm{H} & -2.184199 & -11.504663 & 1.553853 \\ \mathrm{H} & -3.620642 & -10.707460 & 0.830120 \\ \mathrm{H} & -3.629906 & -7.715278 & -2.894097 \\ \mathrm{H} & -3.651509 & -9.460048 & -3.253909 \\ \mathrm{H} & -5.110188 & -8.681746 & -2.561587 \\ \mathrm{H} & -0.423936 & -7.531145 & -2.265076 \\ \mathrm{H} & 0.409188 & -6.378440 & -3.174599 \\ \mathrm{H} & -0.451703 & -2.998618 & -2.025508 \\ \mathrm{H} & -0.544337 & -3.379077 & -0.470816 \\ \mathrm{H} & -2.189133 & -4.471001 & -0.010080 \\ \mathrm{H} & -2.221800 & -6.172232 & -0.206310 \\ \mathrm{H} & 0.580212 & -4.007004 & -3.981352 \\ \mathrm{H} & -0.800720 & -4.504429 & -4.748578 \\ \mathrm{H} & -4.318637 & -3.465262 & -2.055409 \\ \mathrm{H} & -4.430841 & -5.216118 & -2.119919 \\ \mathrm{H} & -5.888173 & -4.228397 & -2.522802 \\ \mathrm{H} & -2.459491 & -5.432183 & -7.210503 \\ \mathrm{H} & -2.500750 & -7.164278 & -6.771201 \\ \mathrm{H} & -3.827315 & -6.468746 & -7.747256 \\ & & & \\ \mathrm{E}(\mathrm{RB}+\mathrm{HF}-\mathrm{LYP})=-3526.9016194 & \\ & & & \end{array}$




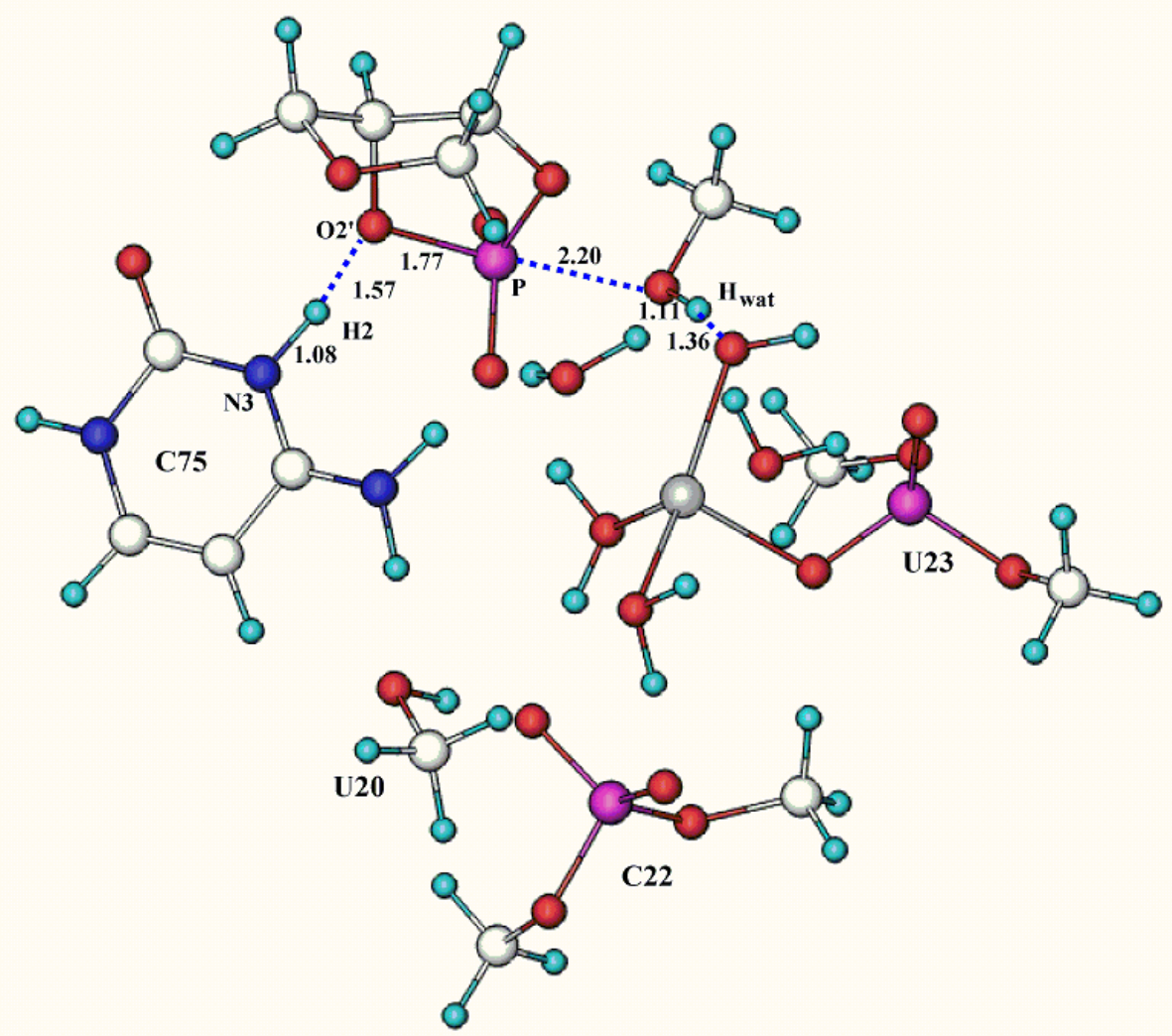

Figure 7. Optimized structure of the TS2 of C75-base mechanism.

Product of C75-base mechanism

b3lyp/3-21g* optimized structure:

$\begin{array}{lrrr}\mathrm{C} & 0.000000 & 0.000000 & 0.000000 \\ \mathrm{~N} & 0.000000 & 0.000000 & 1.381062 \\ \mathrm{C} & 1.146226 & 0.000000 & 2.161119 \\ \mathrm{~N} & 2.345344 & -0.112081 & 1.430363 \\ \mathrm{C} & 2.393595 & -0.216113 & 0.054450 \\ \mathrm{C} & 1.270972 & -0.159132 & -0.683628 \\ \mathrm{O} & 1.171366 & 0.094848 & 3.393058 \\ \mathrm{~N} & -1.088060 & 0.143729 & -0.696529 \\ \mathrm{O} & -0.516957 & -0.153369 & -3.228743 \\ \mathrm{C} & -0.861052 & 1.049542 & -4.013689 \\ \mathrm{O} & -2.328666 & -1.798723 & -4.018808 \\ \mathrm{P} & -3.203625 & -1.198476 & -5.119086 \\ \mathrm{O} & -4.166739 & -2.392298 & -5.629285 \\ \mathrm{C} & -5.280579 & -2.074495 & -6.559377 \\ \mathrm{O} & -3.894995 & 0.128468 & -4.902181\end{array}$




\begin{tabular}{|c|c|c|c|}
\hline $\mathrm{O}$ & -2.239686 & -0.955194 & -6.421885 \\
\hline $\mathrm{C}$ & -1.417571 & -2.099992 & -6.850788 \\
\hline $\mathrm{O}$ & -3.887441 & 0.925200 & -2.440459 \\
\hline $\mathrm{Mg}$ & -4.543970 & -0.683198 & -1.245435 \\
\hline $\mathrm{O}$ & -5.470667 & -1.277072 & -2.918632 \\
\hline $\mathrm{P}$ & -6.978920 & -1.466136 & -3.039570 \\
\hline $\mathrm{O}$ & -7.454646 & -0.885842 & -4.495169 \\
\hline $\mathrm{C}$ & -7.104401 & 0.538992 & -4.731356 \\
\hline $\mathrm{O}$ & -5.778271 & -1.686294 & 0.043652 \\
\hline $\mathrm{O}$ & -3.538863 & 0.278645 & 0.325918 \\
\hline $\mathrm{O}$ & -3.100198 & -2.037959 & -1.449661 \\
\hline $\mathrm{O}$ & -7.802970 & -0.825462 & -1.934253 \\
\hline $\mathrm{O}$ & -7.317229 & -3.020270 & -3.218612 \\
\hline $\mathrm{C}$ & -6.168093 & -3.981641 & -3.227623 \\
\hline $\mathrm{O}$ & -6.126201 & 0.732316 & -0.642454 \\
\hline $\mathrm{O}$ & -4.602761 & -3.783184 & -0.463230 \\
\hline $\mathrm{C}$ & -4.206373 & -4.655954 & 0.642396 \\
\hline $\mathrm{O}$ & -2.389866 & -0.404924 & 2.420001 \\
\hline $\mathrm{P}$ & -2.736753 & -1.488534 & 3.482132 \\
\hline $\mathrm{O}$ & -3.858396 & -0.806797 & 4.481682 \\
\hline $\mathrm{C}$ & -3.205943 & -0.025613 & 5.526297 \\
\hline $\mathrm{C}$ & -2.872095 & 1.418823 & 5.090668 \\
\hline $\mathrm{O}$ & -1.705944 & 1.770820 & 5.906962 \\
\hline $\mathrm{C}$ & -0.839037 & 0.567700 & 5.871689 \\
\hline $\mathrm{C}$ & -1.783699 & -0.652025 & 5.756779 \\
\hline $\mathrm{O}$ & -1.494542 & -1.450611 & 4.559817 \\
\hline $\mathrm{O}$ & -3.099040 & -2.857000 & 3.047655 \\
\hline $\mathrm{H}$ & -6.678199 & -1.777153 & -0.380015 \\
\hline $\mathrm{H}$ & -5.202366 & -2.892827 & -0.101692 \\
\hline $\mathrm{H}$ & -0.944843 & -0.117166 & 1.894142 \\
\hline $\mathrm{H}$ & -3.811100 & -4.084212 & 1.488608 \\
\hline $\mathrm{H}$ & -5.061161 & -5.259741 & 0.983874 \\
\hline $\mathrm{H}$ & -3.432982 & -5.344689 & 0.278835 \\
\hline $\mathrm{H}$ & -3.821910 & -0.052208 & 6.424364 \\
\hline $\mathrm{H}$ & -3.661146 & 2.139142 & 5.310460 \\
\hline $\mathrm{H}$ & -1.749951 & -1.310595 & 6.626570 \\
\hline $\mathrm{H}$ & -0.260798 & 0.573578 & 6.798241 \\
\hline $\mathrm{H}$ & -0.181716 & 0.558971 & 4.998188 \\
\hline $\mathrm{H}$ & -2.644002 & 1.398407 & 4.018678 \\
\hline $\mathrm{H}$ & 3.188064 & -0.130679 & 1.993554 \\
\hline $\mathrm{H}$ & 3.375253 & -0.337544 & -0.383784 \\
\hline $\mathrm{H}$ & 1.236803 & -0.235634 & -1.761020 \\
\hline $\mathrm{H}$ & -2.059668 & 0.240834 & -0.293119 \\
\hline $\mathrm{H}$ & -0.978765 & 0.046412 & -1.759018 \\
\hline
\end{tabular}




\begin{tabular}{lrrr}
$\mathrm{H}$ & -1.167641 & -0.911101 & -3.546791 \\
$\mathrm{H}$ & -0.042023 & 1.764787 & -3.897810 \\
$\mathrm{H}$ & -1.799384 & 1.487271 & -3.660549 \\
$\mathrm{H}$ & -0.975902 & 0.780718 & -5.068874 \\
$\mathrm{H}$ & -0.784120 & -2.439585 & -6.025842 \\
$\mathrm{H}$ & -0.807556 & -1.754321 & -7.686952 \\
$\mathrm{H}$ & -2.057827 & -2.931028 & -7.165322 \\
$\mathrm{H}$ & -6.135621 & -1.716315 & -5.985634 \\
$\mathrm{H}$ & -4.966260 & -1.330646 & -7.299583 \\
$\mathrm{H}$ & -5.522696 & -3.013443 & -7.060572 \\
$\mathrm{H}$ & -3.947685 & 0.601385 & -3.434922 \\
$\mathrm{H}$ & -4.693375 & 1.477838 & -2.259070 \\
$\mathrm{H}$ & -3.963904 & 1.164705 & 0.441604 \\
$\mathrm{H}$ & -3.214856 & -0.107069 & 1.269938 \\
$\mathrm{H}$ & -3.587569 & -2.910383 & -1.080512 \\
$\mathrm{H}$ & -2.865074 & -2.130168 & -2.428438 \\
$\mathrm{H}$ & -6.077641 & 0.006189 & 0.080503 \\
$\mathrm{H}$ & -6.904772 & 0.343540 & -1.203568 \\
$\mathrm{H}$ & -5.704858 & -4.032578 & -2.236340 \\
$\mathrm{H}$ & -5.423056 & -3.668947 & -3.962736 \\
$\mathrm{H}$ & -6.609934 & -4.940824 & -3.503062 \\
$\mathrm{H}$ & -7.404327 & 1.143363 & -3.866889 \\
$\mathrm{H}$ & -6.028037 & 0.616143 & -4.903471 \\
$\mathrm{H}$ & -7.671787 & 0.852889 & -5.609418 \\
& & & \\
$\mathrm{E}(\mathrm{RB}+\mathrm{HF}-\mathrm{LYP})=-3526.9338096$ & \\
\hline & & &
\end{tabular}




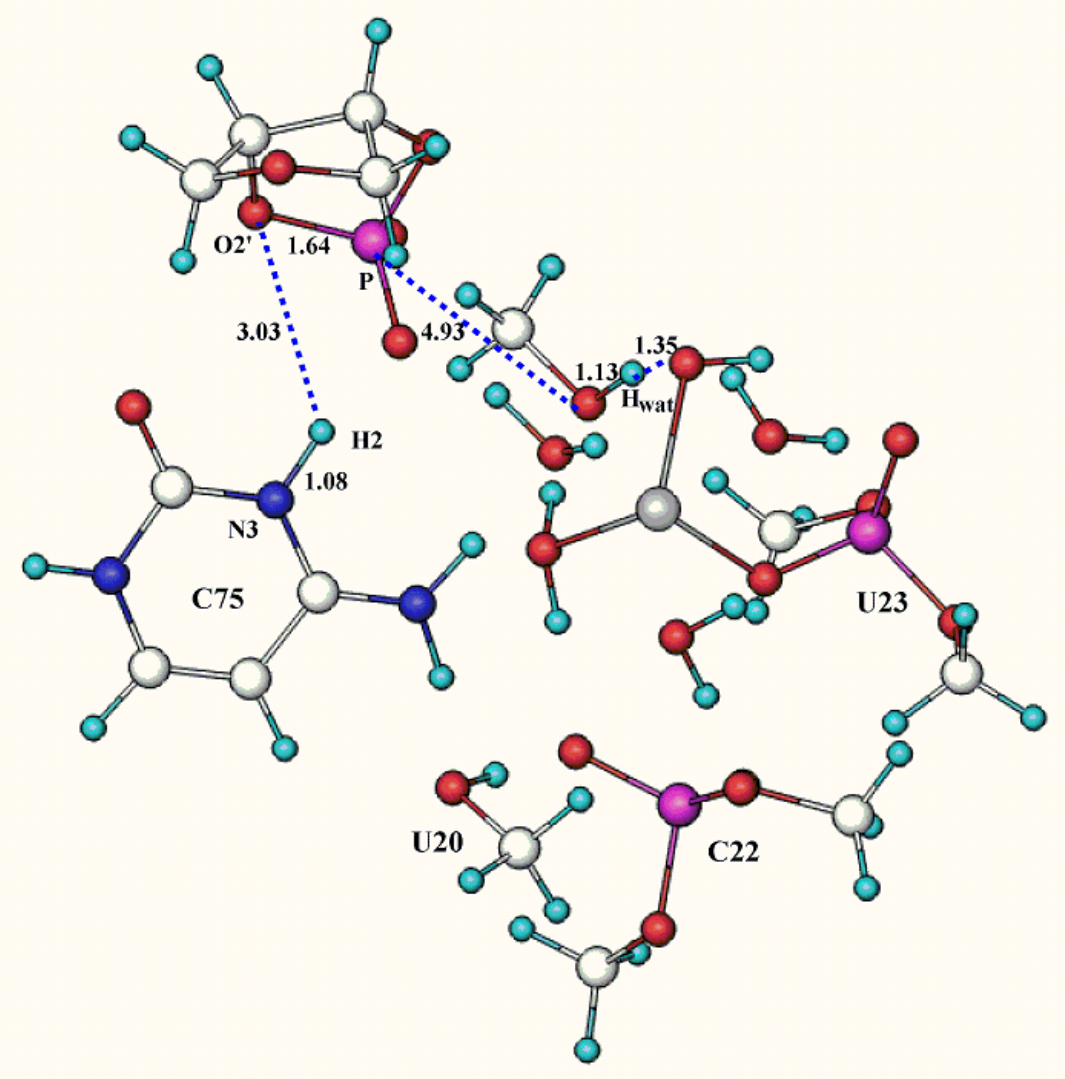

Figure 8. Optimized structure of the Product of C75-base mechanism.

Table 1. Relevant Distance(in $\AA$ ) and Angle(in degree) for the structures in C75-base mechanism.

\begin{tabular}{cccccc}
\hline species & $\mathrm{O} 2^{\prime}-\mathrm{P}$ & $\mathrm{P}-\mathrm{OCH}_{3}$ & $\mathrm{H} 2-\mathrm{N} 3$ & $\mathrm{H}_{\text {wat }}-\mathrm{OCH}_{3}$ & $\mathrm{O}^{\prime}-\mathrm{P}-\mathrm{OCH}_{3}$ \\
\hline reactant & 2.81 & 1.69 & 1.70 & 1.60 & 163.1 \\
\hline TS1 & 1.99 & 1.75 & 1.21 & 1.55 & 158.9 \\
\hline intermediate & 1.93 & 1.77 & 1.15 & 1.53 & 159.3 \\
\hline TS2 & 1.77 & 2.20 & 1.08 & 1.11 & 163.3 \\
\hline product & 1.64 & 4.93 & 1.08 & 1.13 & 145.2 \\
\hline
\end{tabular}


Table 2. Particle Mulliken charges(in e) for the structures in C75-base mechanism

\begin{tabular}{ccccccc}
\hline species & $\mathrm{Mg}^{2+}$ & $\mathrm{O}^{\prime}$ & $\mathrm{P}$ & $\mathrm{N} 3$ & $\mathrm{O}^{\prime}$ & $\mathrm{O}_{\text {wat }}$ \\
\hline reactant & 0.91 & -0.67 & 1.35 & -0.76 & -0.40 & -0.81 \\
\hline TS1 & 1.05 & -0.55 & 0.80 & -0.69 & -0.43 & -0.86 \\
\hline intermediate & 1.07 & -0.52 & 0.75 & -0.68 & -0.41 & -0.87 \\
\hline TS2 & 1.19 & -0.42 & 1.04 & -0.61 & -0.49 & -0.86 \\
\hline product & 0.97 & -0.61 & 0.98 & -0.62 & -0.69 & -0.87 \\
\hline
\end{tabular}

Addition: $\mathbf{O}_{\text {wat }}$ is the oxygen atom of water coordinate with $\mathrm{Mg}^{2+}$ which acts general acid in the C75-base mechanism.

Table 3. summary data for the structures in C75-base mechanism

\begin{tabular}{cccc}
\hline species & $\mathrm{HF}($ Hartrees $)$ & $\mathrm{TCG}($ Hartrees $)$ & $\Delta \mathrm{G}_{\text {solv }}(\mathrm{kcal} / \mathrm{mol})$ \\
\hline reactant & -3526.954021 & 0.5624 & -36.00 \\
\hline TS1 & -3526.914797 & 0.559156 & -39.05 \\
\hline intermediate & -3526.91286 & 0.559514 & -39.49 \\
\hline TS2 & -3526.901619 & 0.56553 & -39.55 \\
\hline product & -3526.93381 & 0.564132 & -40.09 \\
\hline
\end{tabular}

Addition: HF is the single point energy of each species and TCG is Thermal correction to Gibbs Free Energy which contains the Zero point correction. 
Reactant of C75-acid mechanism

b3lyp/3-21g* optimized structure:

$\begin{array}{cccc}\mathrm{N} & 0.000000 & 0.000000 & 0.000000 \\ \mathrm{C} & 0.000000 & 0.000000 & 1.378999 \\ \mathrm{C} & 1.285214 & 0.000000 & 2.050550 \\ \mathrm{C} & 2.400144 & 0.036595 & 1.297002 \\ \mathrm{~N} & 2.335460 & 0.076859 & -0.084896 \\ \mathrm{C} & 1.125793 & 0.053479 & -0.801613 \\ \mathrm{~N} & -1.112529 & -0.006047 & 2.050559 \\ \mathrm{O} & 1.099915 & 0.081905 & -2.039426 \\ \mathrm{O} & -0.521547 & -0.275977 & 4.553905 \\ \mathrm{C} & -1.372163 & -1.414754 & 4.985836 \\ \mathrm{O} & -1.281479 & 1.733039 & 6.022501 \\ \mathrm{P} & -1.937567 & 1.368531 & 7.374933 \\ \mathrm{O} & -0.765144 & 1.084438 & 8.447419 \\ \mathrm{C} & 0.264081 & 2.123813 & 8.605291 \\ \mathrm{O} & -2.881723 & 0.195033 & 7.396763 \\ \mathrm{O} & -2.562451 & 2.725828 & 7.982899 \\ \mathrm{C} & -3.204546 & 3.708435 & 7.077141 \\ \mathrm{O} & -2.863052 & 1.650946 & 4.010455 \\ \mathrm{Mg} & -4.756000 & 1.019975 & 3.997923 \\ \mathrm{O} & -4.985306 & 0.164960 & 5.850286 \\ \mathrm{O} & -4.354525 & -0.789241 & 3.179952 \\ \mathrm{O} & -4.864424 & 2.242155 & 2.282183 \\ \mathrm{O} & -6.704590 & 0.810713 & 3.123188 \\ \mathrm{O} & -5.612419 & 2.530006 & 5.218141 \\ \mathrm{P} & -6.243642 & 3.797231 & 4.645170 \\ \mathrm{O} & -6.673112 & 4.671448 & 5.946378 \\ \mathrm{C} & -6.915976 & 6.110125 & 5.769206 \\ \mathrm{O} & -5.480247 & 4.640716 & 3.658620 \\ \mathrm{O} & -7.664515 & 3.322566 & 3.977032 \\ \mathrm{C} & -8.049409 & 3.933839 & 2.668666 \\ \mathrm{O} & -3.375138 & -0.581126 & 0.862263 \\ \mathrm{P} & -3.744417 & -0.532885 & -0.621932 \\ \mathrm{O} & -2.284434 & -0.106362 & -1.282726 \\ \mathrm{C} & -2.192328 & 0.088647 & -2.742763 \\ \mathrm{O} & -4.552473 & 0.837214 & -0.953828 \\ \mathrm{C} & -5.971532 & 0.770231 & -1.311027 \\ \mathrm{C} & -6.898775 & 1.320830 & -0.201687 \\ \mathrm{C} & -8.169609 & 1.629931 & -1.016032 \\ \mathrm{O} & -7.743741 & 1.847942 & -2.427258 \\ & -6.275672 & 1.757670 & -2.439421 \\ \mathrm{O} & & 2.584230 & 0.267389\end{array}$




\begin{tabular}{|c|c|c|c|}
\hline $\mathrm{O}$ & -4.345305 & -1.710103 & -1.307585 \\
\hline $\mathrm{H}$ & -8.632841 & 2.535519 & -0.617165 \\
\hline $\mathrm{H}$ & -6.233244 & -0.252608 & -1.590359 \\
\hline $\mathrm{H}$ & -7.076268 & 0.612925 & 0.617165 \\
\hline $\mathrm{H}$ & -5.590107 & 2.393513 & 0.950970 \\
\hline $\mathrm{H}$ & -8.878848 & 0.795320 & -1.002889 \\
\hline $\mathrm{H}$ & -5.803475 & 2.707702 & -2.171621 \\
\hline $\mathrm{H}$ & -5.963362 & 1.411523 & -3.426742 \\
\hline $\mathrm{H}$ & -1.125089 & 0.156959 & -2.957599 \\
\hline $\mathrm{H}$ & -2.719696 & 1.006075 & -3.016792 \\
\hline $\mathrm{H}$ & -2.648413 & -0.769552 & -3.244117 \\
\hline $\mathrm{H}$ & -1.833059 & -1.184372 & 5.949251 \\
\hline $\mathrm{H}$ & -0.725642 & 0.527805 & 5.173678 \\
\hline $\mathrm{H}$ & -2.176835 & -1.570878 & 4.258446 \\
\hline $\mathrm{H}$ & -0.748669 & -2.310951 & 5.063206 \\
\hline $\mathrm{H}$ & 1.014530 & 1.719277 & 9.285785 \\
\hline $\mathrm{H}$ & -0.183095 & 3.031107 & 9.022136 \\
\hline $\mathrm{H}$ & 0.713114 & 2.357799 & 7.634437 \\
\hline $\mathrm{H}$ & -2.484673 & 4.043387 & 6.324487 \\
\hline $\mathrm{H}$ & -3.510817 & 4.542262 & 7.709471 \\
\hline $\mathrm{H}$ & -6.691585 & 6.592022 & 6.723404 \\
\hline $\mathrm{H}$ & -4.076580 & 3.280086 & 6.577274 \\
\hline $\mathrm{H}$ & -7.965240 & 6.293654 & 5.508419 \\
\hline $\mathrm{H}$ & -6.269918 & 6.500117 & 4.977741 \\
\hline $\mathrm{H}$ & -9.121041 & 3.752756 & 2.566656 \\
\hline $\mathrm{H}$ & -7.494569 & 3.464409 & 1.848297 \\
\hline $\mathrm{H}$ & -7.840282 & 5.005879 & 2.673551 \\
\hline $\mathrm{H}$ & 3.392901 & 0.029887 & 1.726807 \\
\hline $\mathrm{H}$ & 1.270528 & -0.066413 & 3.128904 \\
\hline $\mathrm{H}$ & -2.053643 & -0.128983 & 1.561875 \\
\hline $\mathrm{H}$ & -1.018939 & -0.011876 & 3.125151 \\
\hline $\mathrm{H}$ & 3.175535 & 0.117476 & -0.650947 \\
\hline $\mathrm{H}$ & -0.939170 & -0.037711 & -0.511210 \\
\hline $\mathrm{H}$ & -5.207079 & -1.287345 & 3.243496 \\
\hline $\mathrm{H}$ & -4.042720 & -0.756930 & 2.184546 \\
\hline $\mathrm{H}$ & -2.861978 & 2.308962 & 3.271608 \\
\hline $\mathrm{H}$ & -2.214065 & 1.841638 & 4.785726 \\
\hline $\mathrm{H}$ & -5.487903 & 0.961701 & 6.182764 \\
\hline $\mathrm{H}$ & -4.158572 & 0.058568 & 6.454709 \\
\hline $\mathrm{H}$ & -4.967016 & 3.198838 & 2.580209 \\
\hline $\mathrm{H}$ & -6.107760 & 1.500320 & 2.550237 \\
\hline $\mathrm{H}$ & -7.334782 & 1.412733 & 3.613110 \\
\hline
\end{tabular}




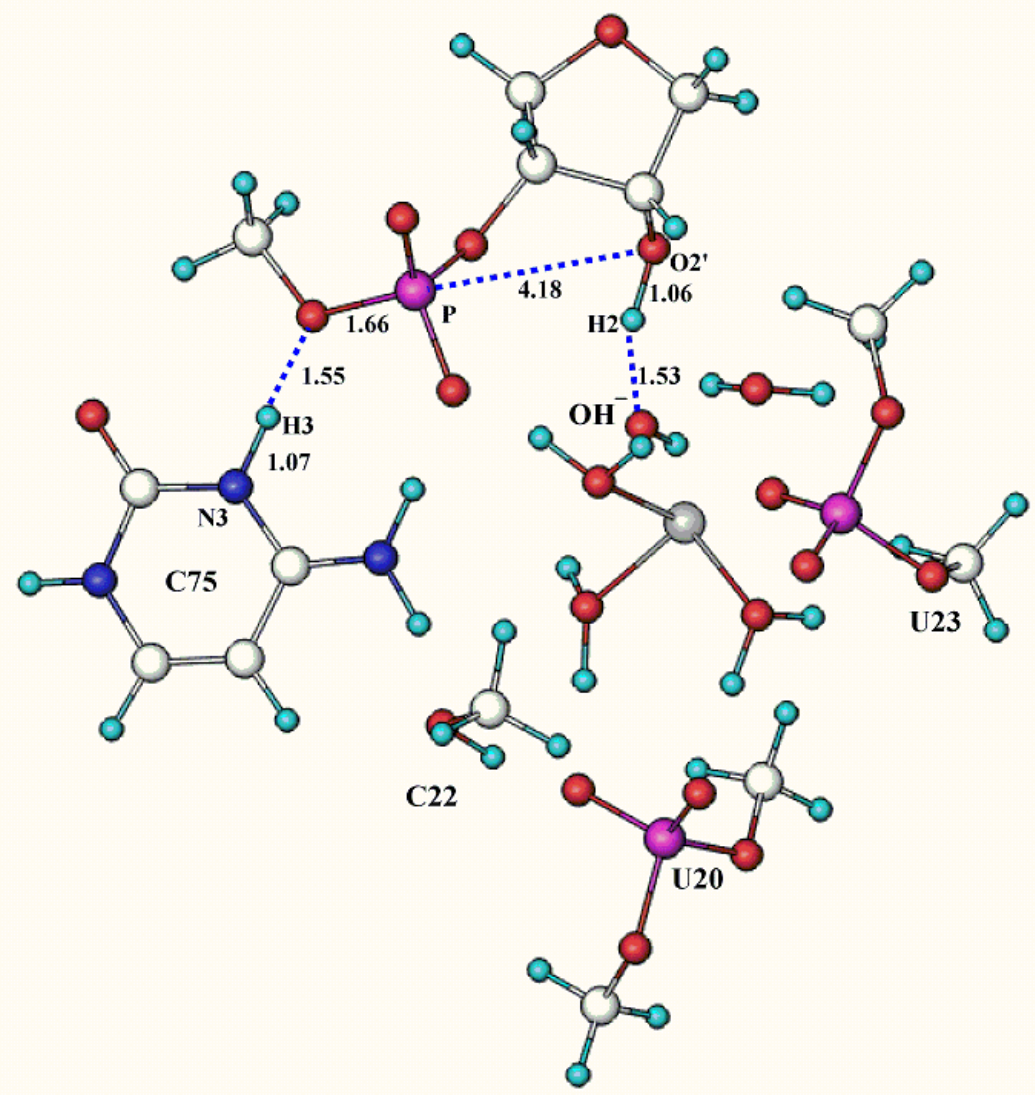

Figure 9. Optimized structure of the Reactant of C75-acid mechanism.

\section{TS1 of C75-acid mechanism}

b3lyp/3-21g* optimized structure:

$\begin{array}{cccc}\mathrm{C} & 0.000000 & 0.000000 & 0.000000 \\ \mathrm{C} & 0.000000 & 0.000000 & 1.581260 \\ \mathrm{C} & 1.491582 & 0.000000 & 1.984977 \\ \mathrm{O} & 2.287021 & 0.148016 & 0.741320 \\ \mathrm{C} & 1.448898 & -0.349080 & -0.362924 \\ \mathrm{O} & -0.655267 & 1.225004 & 2.019124 \\ \mathrm{P} & -1.871804 & 1.772416 & 0.990659 \\ \mathrm{O} & -2.770918 & 0.688990 & 0.481558 \\ \mathrm{O} & -0.370298 & 1.336205 & -0.366116 \\ \mathrm{O} & -1.746743 & 3.226112 & 0.426428 \\ \mathrm{O} & -2.718592 & 2.342872 & 2.399193 \\ \mathrm{C} & -3.189069 & 1.323087 & 3.341055 \\ \mathrm{O} & 1.418027 & 2.845145 & 0.351261 \\ \mathrm{Mg} & 1.413321 & 4.842216 & -0.197371\end{array}$




\begin{tabular}{|c|c|c|c|}
\hline $\mathrm{O}$ & 1.881000 & 6.430778 & -1.321849 \\
\hline $\mathrm{O}$ & -0.279929 & 4.356915 & -1.194936 \\
\hline $\mathrm{O}$ & 0.556163 & 5.839475 & 1.347145 \\
\hline $\mathrm{O}$ & 2.885804 & 3.761807 & -1.376155 \\
\hline $\mathrm{O}$ & 3.017349 & 5.158649 & 1.257420 \\
\hline $\mathrm{P}$ & 3.758131 & 3.984864 & 1.903056 \\
\hline $\mathrm{O}$ & 2.956021 & 2.881499 & 2.548953 \\
\hline $\mathrm{O}$ & 4.864627 & 4.553159 & 2.948406 \\
\hline $\mathrm{C}$ & 4.553749 & 4.488812 & 4.387045 \\
\hline $\mathrm{O}$ & 4.710978 & 3.397601 & 0.712020 \\
\hline $\mathrm{C}$ & 4.996938 & 1.934230 & 0.741670 \\
\hline $\mathrm{O}$ & 1.503563 & 8.823182 & -0.553869 \\
\hline $\mathrm{P}$ & 0.974786 & 9.032808 & 0.845832 \\
\hline $\mathrm{O}$ & 2.055810 & 8.599087 & 1.964259 \\
\hline $\mathrm{C}$ & 3.465686 & 8.317624 & 1.582439 \\
\hline $\mathrm{O}$ & 0.810658 & 10.630585 & 1.058820 \\
\hline $\mathrm{C}$ & 0.377767 & 11.084943 & 2.389587 \\
\hline $\mathrm{O}$ & -0.330374 & 8.346826 & 1.273222 \\
\hline $\mathrm{N}$ & -2.970217 & 4.702905 & 3.345110 \\
\hline $\mathrm{C}$ & -2.759860 & 5.762041 & 2.487757 \\
\hline $\mathrm{C}$ & -2.764534 & 7.098419 & 3.055274 \\
\hline $\mathrm{C}$ & -3.044806 & 7.236342 & 4.365689 \\
\hline $\mathrm{N}$ & -3.342680 & 6.144983 & 5.154603 \\
\hline $\mathrm{C}$ & -3.302811 & 4.815989 & 4.677208 \\
\hline $\mathrm{N}$ & -2.559931 & 5.536715 & 1.221531 \\
\hline $\mathrm{O}$ & -3.566114 & 3.866425 & 5.429219 \\
\hline $\mathrm{O}$ & -2.222104 & 7.660128 & -0.337274 \\
\hline $\mathrm{C}$ & -1.568443 & 7.309537 & -1.618971 \\
\hline $\mathrm{H}$ & 0.585741 & 2.149520 & 0.034508 \\
\hline $\mathrm{H}$ & 1.798664 & 2.626126 & 1.254606 \\
\hline $\mathrm{H}$ & -3.632078 & 1.859897 & 4.182585 \\
\hline $\mathrm{H}$ & -3.909189 & 0.671607 & 2.838913 \\
\hline $\mathrm{H}$ & -2.337382 & 0.729924 & 3.686392 \\
\hline $\mathrm{H}$ & -0.525190 & -0.871256 & 1.982693 \\
\hline $\mathrm{H}$ & 1.754505 & -0.949658 & 2.471018 \\
\hline $\mathrm{H}$ & 1.744781 & 0.849699 & 2.618872 \\
\hline $\mathrm{H}$ & 1.564578 & -1.438677 & -0.473090 \\
\hline $\mathrm{H}$ & 1.764537 & 0.160675 & -1.273999 \\
\hline $\mathrm{H}$ & -0.728472 & -0.710210 & -0.395884 \\
\hline $\mathrm{H}$ & -0.932700 & 3.753517 & -0.535237 \\
\hline $\mathrm{H}$ & -0.172842 & 3.894263 & -2.061655 \\
\hline $\mathrm{H}$ & 2.391554 & 3.054584 & -0.769109 \\
\hline $\mathrm{H}$ & 3.753723 & 3.869953 & -0.884924 \\
\hline $\mathrm{H}$ & 4.066312 & 1.361281 & 0.722384 \\
\hline
\end{tabular}




$\begin{array}{rrrr}\mathrm{H} & 5.570566 & 1.685485 & 1.639922 \\ \mathrm{H} & 5.601240 & 1.741144 & -0.145713 \\ \mathrm{H} & 1.765172 & 7.436853 & -1.075333 \\ \mathrm{H} & 2.606358 & 6.270165 & -1.967069 \\ \mathrm{H} & 0.069274 & 6.731068 & 1.380932 \\ \mathrm{H} & 1.402814 & 5.896672 & 1.872232 \\ \mathrm{H} & 3.911476 & 3.627604 & 4.586026 \\ \mathrm{H} & 5.504436 & 4.395936 & 4.916470 \\ \mathrm{H} & 4.046038 & 5.405928 & 4.706071 \\ \mathrm{H} & 4.104830 & 8.784778 & 2.334961 \\ \mathrm{H} & 3.675201 & 8.726592 & 0.591334 \\ \mathrm{H} & 3.595278 & 7.233987 & 1.570037 \\ \mathrm{H} & -0.580606 & 10.625943 & 2.651948 \\ \mathrm{H} & 1.123198 & 10.808171 & 3.142557 \\ \mathrm{H} & 0.278186 & 12.169607 & 2.332508 \\ \mathrm{H} & -1.355597 & 6.236674 & -1.655065 \\ \mathrm{H} & -0.616084 & 7.844774 & -1.707452 \\ \mathrm{H} & -2.232778 & 7.601236 & -2.439842 \\ \mathrm{H} & -1.496778 & 8.079344 & 0.254711 \\ \mathrm{H} & -2.453013 & 6.363284 & 0.547663 \\ \mathrm{H} & -2.340398 & 4.524208 & 0.887590 \\ \mathrm{H} & -2.854303 & 3.675858 & 2.928734 \\ \mathrm{H} & -3.576007 & 6.245485 & 6.135680 \\ \mathrm{H} & -3.035252 & 8.198353 & 4.861265 \\ \mathrm{H} & -2.438905 & 7.923598 & 2.443088 \\ & & & \\ \mathrm{E}(\mathrm{RB}+\mathrm{HF}-\mathrm{LYP})=-3526.913468 & \\ & & & \end{array}$




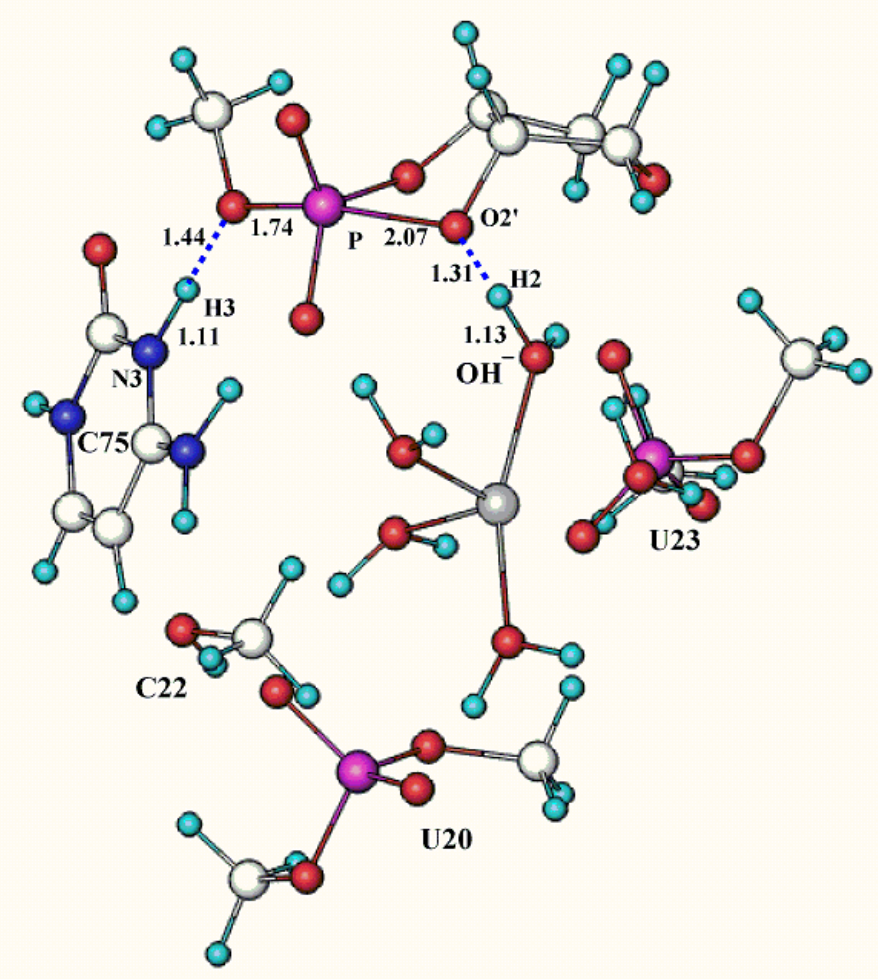

Figure 10. Optimized structure of the TS1 of C75-acid mechanism.

\section{Intermediate of C75-acid mechanism}

b3lyp/3-21g* optimized structure:

$\begin{array}{cccc}\mathrm{C} & 0.000000 & 0.000000 & 0.000000 \\ \mathrm{C} & 0.000000 & 0.000000 & 1.571305 \\ \mathrm{C} & 1.502520 & 0.000000 & 1.960499 \\ \mathrm{O} & 2.294748 & -0.045572 & 0.704292 \\ \mathrm{C} & 1.394837 & -0.496534 & -0.371418 \\ \mathrm{O} & -0.669611 & 1.211053 & 2.013274 \\ \mathrm{P} & -1.651717 & 1.964565 & 0.862218 \\ \mathrm{O} & -2.684141 & 1.081200 & 0.222867 \\ \mathrm{O} & -0.208164 & 1.381696 & -0.336048 \\ \mathrm{O} & -1.264290 & 3.418222 & 0.413058 \\ \mathrm{O} & -2.552841 & 2.609922 & 2.234517 \\ \mathrm{C} & -3.249709 & 1.612751 & 3.058979 \\ \mathrm{O} & 0.154340 & 4.442805 & -1.308480 \\ \mathrm{Mg} & 1.999460 & 4.678878 & -0.540794 \\ \mathrm{O} & 2.517150 & 6.170682 & -1.773928\end{array}$




\begin{tabular}{|c|c|c|c|}
\hline $\mathrm{O}$ & 1.464690 & 5.761239 & 1.080202 \\
\hline $\mathrm{O}$ & 1.816713 & 2.706126 & 0.116263 \\
\hline $\mathrm{O}$ & 3.155794 & 3.363460 & -1.831299 \\
\hline $\mathrm{O}$ & 3.823115 & 4.817610 & 0.653106 \\
\hline $\mathrm{P}$ & 4.455999 & 3.583981 & 1.299805 \\
\hline $\mathrm{O}$ & 3.578514 & 2.659042 & 2.109500 \\
\hline $\mathrm{O}$ & 5.753917 & 4.025597 & 2.170044 \\
\hline $\mathrm{C}$ & 5.604134 & 4.118026 & 3.633370 \\
\hline $\mathrm{O}$ & 5.155406 & 2.783658 & 0.058198 \\
\hline $\mathrm{C}$ & 5.236622 & 1.299959 & 0.180263 \\
\hline $\mathrm{O}$ & 2.439377 & 8.603702 & -1.044117 \\
\hline $\mathrm{P}$ & 2.163487 & 8.869495 & 0.419520 \\
\hline $\mathrm{O}$ & 3.363766 & 8.336378 & 1.357880 \\
\hline $\mathrm{C}$ & 4.655391 & 7.898763 & 0.767531 \\
\hline $\mathrm{O}$ & 2.182732 & 10.475699 & 0.624147 \\
\hline $\mathrm{C}$ & 1.937560 & 10.973366 & 1.988933 \\
\hline $\mathrm{O}$ & 0.887730 & 8.315925 & 1.064026 \\
\hline $\mathrm{N}$ & -2.128150 & 5.736514 & 1.023530 \\
\hline $\mathrm{C}$ & -3.131559 & 5.935457 & 1.824574 \\
\hline $\mathrm{N}$ & -3.671943 & 4.887270 & 2.536201 \\
\hline $\mathrm{C}$ & -4.724414 & 5.005471 & 3.416858 \\
\hline $\mathrm{N}$ & -5.246955 & 6.316315 & 3.532046 \\
\hline $\mathrm{C}$ & -4.749768 & 7.397202 & 2.831172 \\
\hline $\mathrm{C}$ & -3.712387 & 7.256802 & 1.985090 \\
\hline $\mathrm{O}$ & -5.211881 & 4.078638 & 4.080296 \\
\hline $\mathrm{O}$ & -1.364371 & 7.987079 & -0.116597 \\
\hline $\mathrm{C}$ & -0.908033 & 7.649764 & -1.486299 \\
\hline $\mathrm{H}$ & 0.900590 & 2.120670 & -0.044425 \\
\hline $\mathrm{H}$ & 2.299686 & 2.480588 & 0.972340 \\
\hline $\mathrm{H}$ & -4.016859 & 2.147171 & 3.624393 \\
\hline $\mathrm{H}$ & -3.699989 & 0.872501 & 2.391235 \\
\hline $\mathrm{H}$ & -2.535257 & 1.125078 & 3.727845 \\
\hline $\mathrm{H}$ & -0.510254 & -0.878426 & 1.977531 \\
\hline $\mathrm{H}$ & 1.746911 & -0.880689 & 2.567848 \\
\hline $\mathrm{H}$ & 1.779802 & 0.915421 & 2.482657 \\
\hline $\mathrm{H}$ & 1.402209 & -1.594652 & -0.447650 \\
\hline $\mathrm{H}$ & 1.743688 & -0.049908 & -1.303554 \\
\hline $\mathrm{H}$ & -0.810084 & -0.607409 & -0.408773 \\
\hline $\mathrm{H}$ & -0.491153 & 3.875363 & -0.604511 \\
\hline $\mathrm{H}$ & 0.078396 & 4.071893 & -2.220558 \\
\hline $\mathrm{H}$ & 2.652961 & 2.748716 & -1.144466 \\
\hline $\mathrm{H}$ & 4.084750 & 3.359987 & -1.453605 \\
\hline $\mathrm{H}$ & 4.247701 & 0.876816 & 0.373980 \\
\hline $\mathrm{H}$ & 5.922234 & 1.032092 & 0.990414 \\
\hline
\end{tabular}




$\begin{array}{lrrr}\mathrm{H} & 5.639623 & 0.952727 & -0.772076 \\ \mathrm{H} & 2.507282 & 7.188899 & -1.541490 \\ \mathrm{H} & 3.206643 & 5.939771 & -2.437169 \\ \mathrm{H} & 1.087970 & 6.707472 & 1.157389 \\ \mathrm{H} & 2.364121 & 5.732834 & 1.507859 \\ \mathrm{H} & 4.875820 & 3.378757 & 3.975384 \\ \mathrm{H} & 6.587481 & 3.925939 & 4.067490 \\ \mathrm{H} & 5.268447 & 5.121616 & 3.917194 \\ \mathrm{H} & 5.454017 & 8.318063 & 1.383804 \\ \mathrm{H} & 4.737589 & 8.255412 & -0.262010 \\ \mathrm{H} & 4.671783 & 6.807730 & 0.784695 \\ \mathrm{H} & 1.001024 & 10.561159 & 2.375810 \\ \mathrm{H} & 2.758123 & 10.675504 & 2.650309 \\ \mathrm{H} & 1.881101 & 12.060411 & 1.919266 \\ \mathrm{H} & -0.813764 & 6.562947 & -1.593982 \\ \mathrm{H} & 0.076771 & 8.093882 & -1.666831 \\ \mathrm{H} & -1.633527 & 8.045725 & -2.204042 \\ \mathrm{H} & -0.510219 & 8.239709 & 0.408982 \\ \mathrm{H} & -1.752223 & 6.597689 & 0.519728 \\ \mathrm{H} & -1.738880 & 4.727610 & 0.845608 \\ \mathrm{H} & -3.187291 & 3.877266 & 2.392321 \\ \mathrm{H} & -6.023026 & 6.413375 & 4.176524 \\ \mathrm{H} & -5.240918 & 8.346613 & 3.000945 \\ \mathrm{H} & -3.287311 & 8.058292 & 1.399023 \\ & & & \\ \mathrm{E}(\mathrm{RB}+\mathrm{HF}-\mathrm{LYP})=-3526.91515 & \\ & & & \end{array}$




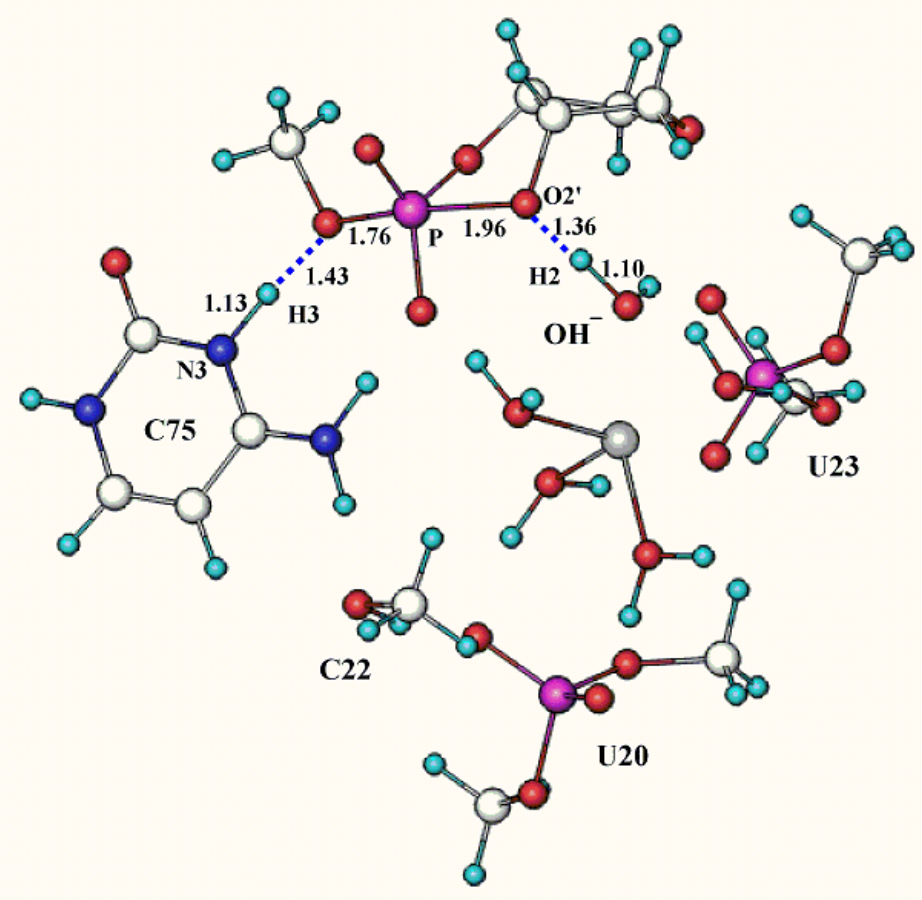

Figure 10. Optimized structure of the Intermediate of C75-acid mechanism.

\section{TS2 of C75-acid mechanism}

b3lyp/3-21g* optimized structure:

$\begin{array}{cccc}\mathrm{N} & 0.000000 & 0.000000 & 0.000000 \\ \mathrm{C} & 0.000000 & 0.000000 & 1.351354 \\ \mathrm{C} & 1.226349 & 0.000000 & 2.124644 \\ \mathrm{C} & 2.390368 & -0.044229 & 1.441442 \\ \mathrm{~N} & 2.386363 & -0.086956 & 0.073185 \\ \mathrm{C} & 1.184870 & -0.059795 & -0.720622 \\ \mathrm{~N} & -1.165755 & -0.035227 & 2.023580 \\ \mathrm{O} & 1.301536 & -0.105336 & -1.948693 \\ \mathrm{O} & -3.425216 & -0.887862 & 0.951192 \\ \mathrm{Mg} & -4.871501 & -0.173311 & 2.136242 \\ \mathrm{O} & -6.111445 & -1.729491 & 1.648317 \\ \mathrm{O} & -6.505983 & 0.639725 & 3.297148 \\ \mathrm{P} & -6.947556 & 1.459233 & 2.094975 \\ \mathrm{O} & -8.528879 & 1.213446 & 1.900912 \\ \mathrm{C} & -9.213611 & 1.669412 & 0.673762\end{array}$




\begin{tabular}{|c|c|c|c|}
\hline $\mathrm{O}$ & -4.273631 & -1.325477 & 3.716164 \\
\hline $\mathrm{O}$ & -3.668347 & 1.393928 & 2.674413 \\
\hline $\mathrm{O}$ & -6.856443 & 3.064730 & 2.354441 \\
\hline $\mathrm{C}$ & -5.703236 & 3.770001 & 1.722594 \\
\hline $\mathrm{O}$ & -6.118726 & 1.115631 & 0.859886 \\
\hline $\mathrm{O}$ & -3.870593 & -0.743463 & -1.398023 \\
\hline $\mathrm{P}$ & -3.651768 & 0.375620 & -2.474959 \\
\hline $\mathrm{O}$ & -2.456617 & 0.542002 & -3.337712 \\
\hline $\mathrm{O}$ & -4.896994 & -0.279566 & -3.475865 \\
\hline $\mathrm{C}$ & -5.966073 & 0.592408 & -3.839463 \\
\hline $\mathrm{C}$ & -7.320427 & 0.012676 & -3.376123 \\
\hline $\mathrm{O}$ & -7.562969 & 0.563358 & -2.018325 \\
\hline $\mathrm{C}$ & -6.987614 & 1.933376 & -2.019678 \\
\hline $\mathrm{C}$ & -5.790060 & 1.900422 & -2.976861 \\
\hline $\mathrm{O}$ & -4.574437 & 1.754370 & -2.221155 \\
\hline $\mathrm{O}$ & -2.380426 & 1.268160 & -0.787273 \\
\hline $\mathrm{C}$ & -1.861648 & 2.567371 & -1.240936 \\
\hline $\mathrm{O}$ & -6.271068 & -1.476978 & -0.817249 \\
\hline $\mathrm{O}$ & -3.937166 & -0.236510 & 6.003061 \\
\hline $\mathrm{P}$ & -3.343386 & 1.146090 & 6.154588 \\
\hline $\mathrm{O}$ & -3.783642 & 1.708921 & 7.610125 \\
\hline $\mathrm{C}$ & -2.890987 & 2.627210 & 8.334630 \\
\hline $\mathrm{O}$ & -4.126369 & 2.185672 & 5.138035 \\
\hline $\mathrm{C}$ & -5.557075 & 2.497189 & 5.431751 \\
\hline $\mathrm{O}$ & -1.868717 & 1.391266 & 5.933553 \\
\hline $\mathrm{O}$ & -0.553969 & -0.347510 & 4.628710 \\
\hline $\mathrm{C}$ & -0.940337 & -1.581707 & 5.317597 \\
\hline $\mathrm{H}$ & -5.309032 & -1.313975 & -1.177320 \\
\hline $\mathrm{H}$ & -6.772740 & -0.628483 & -1.031082 \\
\hline $\mathrm{H}$ & -1.155291 & 2.975560 & -0.508934 \\
\hline $\mathrm{H}$ & -1.385946 & 2.448271 & -2.218297 \\
\hline $\mathrm{H}$ & -2.732081 & 3.215677 & -1.335095 \\
\hline $\mathrm{H}$ & -5.739479 & 2.802947 & -3.592500 \\
\hline $\mathrm{H}$ & -7.750081 & 2.647359 & -2.357058 \\
\hline $\mathrm{H}$ & -6.650451 & 2.108498 & -1.000271 \\
\hline $\mathrm{H}$ & -8.137123 & 0.314706 & -4.043993 \\
\hline $\mathrm{H}$ & -7.253094 & -1.068317 & -3.263834 \\
\hline $\mathrm{H}$ & -5.972748 & 0.807623 & -4.914927 \\
\hline $\mathrm{H}$ & -3.537187 & -0.733717 & -0.231996 \\
\hline $\mathrm{H}$ & -3.345388 & -1.856382 & 1.148696 \\
\hline $\mathrm{H}$ & -6.226809 & -1.678061 & 0.529493 \\
\hline $\mathrm{H}$ & -6.984538 & -1.483370 & 2.051861 \\
\hline $\mathrm{H}$ & -8.846981 & 1.117306 & -0.195164 \\
\hline $\mathrm{H}$ & -9.058596 & 2.744299 & 0.528036 \\
\hline
\end{tabular}




\begin{tabular}{rrrr}
$\mathrm{H}$ & -10.274577 & 1.471838 & 0.833225 \\
$\mathrm{H}$ & -4.184740 & -0.906289 & 4.670585 \\
$\mathrm{H}$ & -4.967129 & -2.030996 & 3.720650 \\
$\mathrm{H}$ & -2.703625 & 1.275086 & 2.442503 \\
$\mathrm{H}$ & -3.805323 & 1.781375 & 3.610293 \\
$\mathrm{H}$ & -4.775688 & 3.243224 & 1.951259 \\
$\mathrm{H}$ & -5.832169 & 3.809416 & 0.639364 \\
$\mathrm{H}$ & -5.708210 & 4.773348 & 2.149536 \\
$\mathrm{H}$ & -5.592266 & 3.132895 & 6.318169 \\
$\mathrm{H}$ & -6.109954 & 1.571689 & 5.587081 \\
$\mathrm{H}$ & -5.956574 & 2.990423 & 4.549678 \\
$\mathrm{H}$ & -1.881467 & 2.557097 & 7.924212 \\
$\mathrm{H}$ & -3.257032 & 3.655635 & 8.240907 \\
$\mathrm{H}$ & -2.902660 & 2.332530 & 9.386810 \\
$\mathrm{H}$ & -0.813793 & -2.408846 & 4.613314 \\
$\mathrm{H}$ & -1.986155 & -1.535571 & 5.636571 \\
$\mathrm{H}$ & -0.302156 & -1.767998 & 6.194178 \\
$\mathrm{H}$ & -0.987916 & 0.437294 & 5.163590 \\
$\mathrm{H}$ & -1.069419 & -0.228276 & 3.054757 \\
$\mathrm{H}$ & -2.033665 & -0.356376 & 1.508392 \\
$\mathrm{H}$ & -1.552236 & 0.661205 & -0.638890 \\
$\mathrm{H}$ & 3.245265 & -0.129933 & -0.464514 \\
$\mathrm{H}$ & 3.355789 & -0.071064 & 1.932588 \\
$\mathrm{H}$ & 1.147995 & -0.018818 & 3.202083 \\
& & & \\
$\mathrm{E}(\mathrm{RB}+\mathrm{HF}-\mathrm{LYP})=-3526.915437$ & \\
\hline & & &
\end{tabular}




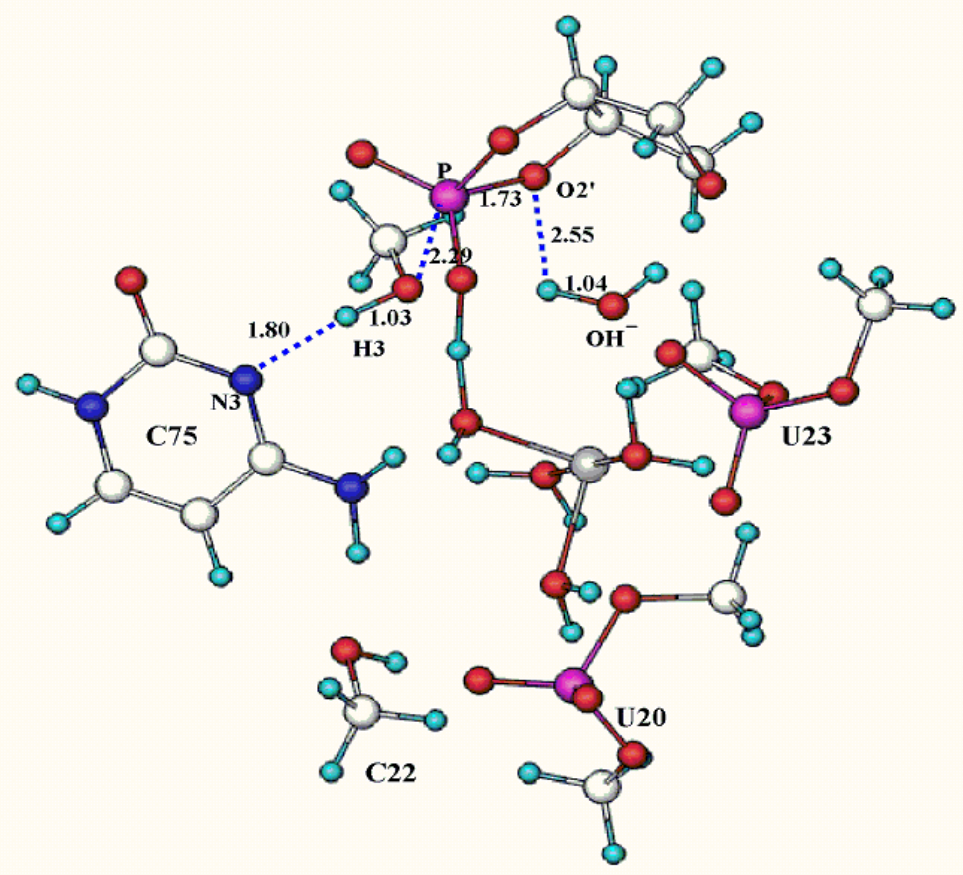

Figure 11. Optimized structure of the TS2 of C75-acid mechanism.

Product of C75-acid mechanism

b31yp/3-21g* optimized structure:

$\begin{array}{lrrr}\mathrm{C} & 0.000000 & 0.000000 & 0.000000 \\ \mathrm{C} & 0.000000 & 0.000000 & 1.450235 \\ \mathrm{~N} & 1.130031 & 0.000000 & 2.168924 \\ \mathrm{C} & 2.365857 & -0.046890 & 1.557955 \\ \mathrm{~N} & 2.357874 & -0.039404 & 0.124497 \\ \mathrm{C} & 1.202762 & -0.014885 & -0.614796 \\ \mathrm{~N} & -1.195768 & -0.004616 & 2.076718 \\ \mathrm{O} & 3.462269 & -0.102590 & 2.138092 \\ \mathrm{O} & -3.044878 & 0.033529 & 0.134924 \\ \mathrm{C} & -4.256216 & 0.855692 & 0.162317 \\ \mathrm{O} & -3.981122 & -2.328199 & 0.090466 \\ \mathrm{P} & -4.971190 & -2.703437 & 1.170133 \\ \mathrm{O} & -6.053625 & -3.759046 & 0.583284 \\ \mathrm{C} & -5.785268 & -4.441781 & -0.691643 \\ \mathrm{O} & -5.730544 & -1.634272 & 1.923323 \\ \mathrm{O} & -4.134777 & -3.645043 & 2.237779 \\ \mathrm{C} & -4.896233 & -4.567836 & 3.127594\end{array}$




\begin{tabular}{|c|c|c|c|}
\hline $\mathrm{O}$ & -4.495120 & -0.108424 & 3.563927 \\
\hline $\mathrm{Mg}$ & -3.217634 & -0.998366 & 4.893139 \\
\hline $\mathrm{O}$ & -2.436651 & -2.143879 & 6.548539 \\
\hline $\mathrm{P}$ & -3.561861 & -3.163273 & 6.359424 \\
\hline $\mathrm{O}$ & -4.362766 & -3.513053 & 7.711763 \\
\hline $\mathrm{C}$ & -3.626991 & -3.876716 & 8.941845 \\
\hline $\mathrm{O}$ & -1.880001 & 0.507556 & 4.699296 \\
\hline $\mathrm{O}$ & -2.351622 & -2.185167 & 3.495349 \\
\hline $\mathrm{O}$ & -4.516280 & -0.003011 & 6.181958 \\
\hline $\mathrm{O}$ & -4.582521 & -2.656314 & 5.350205 \\
\hline $\mathrm{O}$ & -2.909633 & -4.593927 & 5.952273 \\
\hline $\mathrm{C}$ & -1.515858 & -4.567886 & 5.410203 \\
\hline $\mathrm{O}$ & -0.889248 & 1.184138 & 6.816902 \\
\hline $\mathrm{P}$ & 0.482361 & 1.103206 & 7.524903 \\
\hline $\mathrm{O}$ & 0.057876 & 1.339294 & 9.127575 \\
\hline $\mathrm{C}$ & 0.143330 & 0.167457 & 9.969718 \\
\hline $\mathrm{C}$ & 0.639180 & -1.023780 & 9.053278 \\
\hline $\mathrm{C}$ & -0.533020 & -2.016704 & 9.000714 \\
\hline $\mathrm{O}$ & -1.727277 & -1.296988 & 9.517056 \\
\hline $\mathrm{C}$ & -1.247175 & -0.267222 & 10.465487 \\
\hline $\mathrm{O}$ & 0.925447 & -0.472141 & 7.748109 \\
\hline $\mathrm{O}$ & 1.608236 & 1.978261 & 7.120829 \\
\hline $\mathrm{O}$ & -3.029340 & 0.535588 & 8.081652 \\
\hline $\mathrm{O}$ & 0.983523 & -0.303945 & 4.983917 \\
\hline $\mathrm{C}$ & 2.405848 & -0.564895 & 5.259055 \\
\hline $\mathrm{H}$ & -2.197807 & 0.959214 & 7.625129 \\
\hline $\mathrm{H}$ & -2.678287 & -0.339842 & 8.442091 \\
\hline $\mathrm{H}$ & 2.989245 & -0.522579 & 4.333021 \\
\hline $\mathrm{H}$ & 2.775580 & 0.191916 & 5.955658 \\
\hline $\mathrm{H}$ & 2.521996 & -1.553789 & 5.720671 \\
\hline $\mathrm{H}$ & 1.544828 & -1.494139 & 9.442716 \\
\hline $\mathrm{H}$ & -0.337224 & -2.885398 & 9.641805 \\
\hline $\mathrm{H}$ & -0.781752 & -2.295128 & 7.977563 \\
\hline $\mathrm{H}$ & -1.195132 & -0.686783 & 11.477477 \\
\hline $\mathrm{H}$ & -1.953550 & 0.559332 & 10.396513 \\
\hline $\mathrm{H}$ & 0.822640 & 0.359308 & 10.804850 \\
\hline $\mathrm{H}$ & -1.236833 & 0.740375 & 5.572743 \\
\hline $\mathrm{H}$ & -2.398446 & 1.329604 & 4.500742 \\
\hline $\mathrm{H}$ & -3.935063 & 0.280053 & 7.087968 \\
\hline $\mathrm{H}$ & -5.097132 & -0.778949 & 6.403408 \\
\hline $\mathrm{H}$ & -3.102708 & -3.002067 & 9.333112 \\
\hline $\mathrm{H}$ & -2.918047 & -4.685355 & 8.731893 \\
\hline $\mathrm{H}$ & -4.380822 & -4.224849 & 9.649594 \\
\hline $\mathrm{H}$ & -5.051114 & -0.718396 & 2.923667 \\
\hline
\end{tabular}




$\begin{array}{rrrr}\mathrm{H} & -5.066004 & 0.249352 & 4.297530 \\ \mathrm{H} & -1.762765 & -1.667404 & 2.866409 \\ \mathrm{H} & -2.992395 & -2.799372 & 2.985177 \\ \mathrm{H} & -1.454383 & -3.853816 & 4.587684 \\ \mathrm{H} & -0.815241 & -4.271778 & 6.192944 \\ \mathrm{H} & -1.318085 & -5.587468 & 5.078251 \\ \mathrm{H} & -5.262503 & -5.401066 & 2.525031 \\ \mathrm{H} & -5.716653 & -4.032112 & 3.602702 \\ \mathrm{H} & -4.202081 & -4.888499 & 3.900648 \\ \mathrm{H} & -5.016098 & -3.897867 & -1.244033 \\ \mathrm{H} & -5.448399 & -5.467201 & -0.502823 \\ \mathrm{H} & -6.721945 & -4.465758 & -1.254042 \\ \mathrm{H} & -3.995657 & 1.821181 & 0.604781 \\ \mathrm{H} & -5.030736 & 0.379227 & 0.771017 \\ \mathrm{H} & -4.646559 & 1.028572 & -0.851560 \\ \mathrm{H} & -3.359779 & -0.959235 & 0.039819 \\ \mathrm{H} & -2.042661 & 0.076852 & 1.455152 \\ \mathrm{H} & -1.262821 & 0.229879 & 3.090335 \\ \mathrm{H} & 0.948882 & -0.084651 & 3.996944 \\ \mathrm{H} & 3.273021 & -0.067214 & -0.312116 \\ \mathrm{H} & 1.309649 & -0.003614 & -1.692577 \\ \mathrm{H} & -0.949671 & 0.040530 & -0.513953 \\ & & & \\ \mathrm{E}(\mathrm{RB}+\mathrm{HF}-\mathrm{LYP})=-3526.937093 & \end{array}$




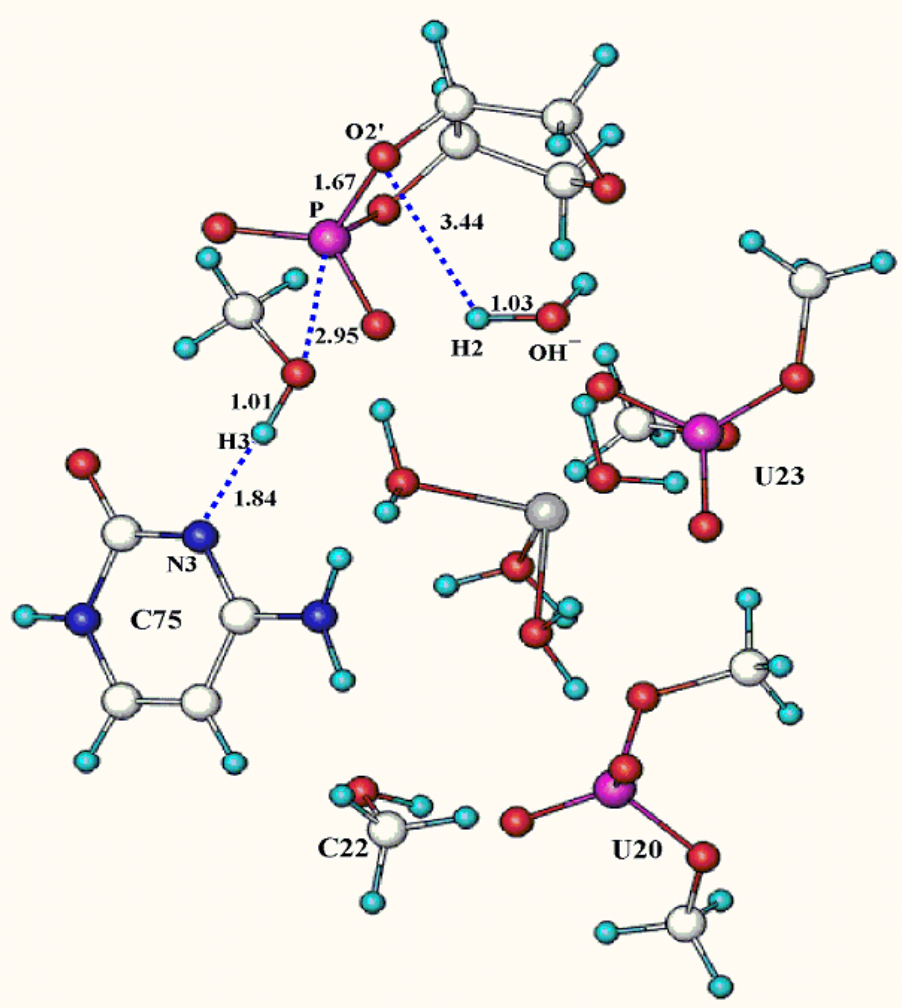

Figure 11. Optimized structure of the Product of C75-acid mechanism.

Table 4. Relevant Distance(in $\AA$ ) and Angle(in degree) for the structures in C75-acid mechanism.

\begin{tabular}{cccccc}
\hline species & $\mathrm{O}^{\prime}-\mathrm{P}$ & $\mathrm{P}^{\prime} \mathrm{OCH}_{3}$ & $\mathrm{H} 2-\mathrm{OH}^{-}$ & $\mathrm{H} 3-\mathrm{OCH}_{3}$ & $\mathrm{O}^{\prime}-\mathrm{P}^{-}-\mathrm{OCH}_{3}$ \\
\hline reactant & 4.18 & 1.66 & 1.53 & 1.55 & 116.6 \\
\hline TS1 & 2.07 & 1.74 & 1.13 & 1.44 & 162.3 \\
\hline intermediate & 1.96 & 1.76 & 1.10 & 1.42 & 163.4 \\
\hline TS2 & 1.73 & 2.29 & 1.04 & 1.03 & 166.8 \\
\hline product & 1.67 & 2.95 & 1.03 & 1.01 & 159.2 \\
\hline
\end{tabular}


Table 5. Particle Mulliken charges(in e) for the structures in C75-acid mechanism

\begin{tabular}{ccccccc}
\hline species & $\mathrm{Mg}^{2+}$ & $\mathrm{O}^{\prime}$ & $\mathrm{P}$ & $\mathrm{N} 3$ & $\mathrm{O}^{\prime}$ & $\mathrm{OH}^{\square}$ \\
\hline reactant & 1.39 & -0.53 & 1.07 & -0.73 & -0.52 & -0.83 \\
\hline TS1 & 1.12 & -0.49 & 0.97 & -0.69 & -0.34 & -0.81 \\
\hline intermediate & 1.29 & -0.41 & 1.04 & -0.73 & -0.42 & -0.95 \\
\hline TS2 & 0.90 & -0.39 & 1.06 & -0.81 & -0.29 & -0.66 \\
\hline product & 0.77 & -0.54 & 1.38 & -0.79 & -0.74 & -0.69 \\
\hline
\end{tabular}

Table 6. summary data for the structures in C75-acid mechanism

\begin{tabular}{cccc}
\hline species & $\mathrm{HF}($ Hartrees $)$ & $\mathrm{TCG}($ Hartrees $)$ & $\Delta \mathrm{G}_{\text {solv }}(\mathrm{kcal} / \mathrm{mol})$ \\
\hline reactant & -3526.941485 & 0.560887 & -37.55 \\
\hline TS1 & -3526.913468 & 0.559378 & -39.6 \\
\hline intermediate & -3526.91515 & 0.559285 & -42.44 \\
\hline TS2 & -3526.915437 & 0.56085 & -39.43 \\
\hline product & -3526.937093 & 0.561444 & -38.08 \\
\hline
\end{tabular}

Addition: HF is the single point energy of each species and TCG is Thermal correction to Gibbs Free Energy which contains the Zero point correction. 